\title{
EXPORTACIÓN DEL ACEITE DE OLIVA. EMPRESAS Y EMPRESARIOS, 1900-1936
}

\author{
Salvador HERNÁNDEZ ARMENTEROS*
}

\section{INTRODUCCIÓN}

Los avances de la Revolución Industrial en el último tercio del siglo XIX trajeron consigo trascendentales innovaciones en todos los sectores de la economía. Desde la agricultura a la industria, pasando por el transporte y demás servicios, experimentaron cambios profundos que afectaron de lleno a uno de los cultivos e industrias derivadas que más se habían extendido en las décadas centrales del siglo, el olivar y la industria aceitera. Mercados como el lubricado de maquinarias, el alumbrado o la fabricación de jabón, entre otros, se fueron perdiendo en un corto período de tiempo. Son las consecuencias, para el sector aceitero, de lo que conocemos como la Crisis agraria finisecular.

Sin embargo, esos mismos avances abrieron otras posibilidades, otros mercados, que hasta ahora eran desconocidos o de una estrechez tal que los hacia despreciables para dicha industria. Este es el caso de las conservas de pescado, entre cuyos inputs el aceite de oliva ocupó un lugar de suma importancia. Para penetrar en este mercado, el sector aceitero tuvo que sufrir, como ocurriera en el resto de los mercados relacionados con el consumo humano, una profunda transformación que, aunque en unos países se adelantó más que en otros, ocupó lo que restaba del siglo XIX y los primeros lustros del XX.

El objetivo de este trabajo es aproximarnos al significado que para la industria aceitera española tuvo este nuevo mercado y cual fue la actitud del empresariado español del sector ante esta demanda.

El análisis lo llevaremos a cabo relacionando España con el resto de los países productores y exportadores de aceite de oliva. Intentaremos, al mismo tiempo, establecer diferencias entre las principales regiones productoras de nuestro país, así como entre las empresas y empresarios que canalizaron este comercio. Para alcanzar estos objetivos Noruega presenta unas condiciones singularmente apro-

* Departamento de Economía Aplicada. Área de Historia e Instituciones Económicas. Universidad de Granada. 
piadas. La importancia de la industria conservera en este país ${ }^{1}$, la estrategia de $\mathrm{s}$ empresas de considerar al aceite de oliva como el mejor conservante y la rique de las fuentes, nos va a permitir un adecuado seguimiento de las exportaciones. su vez, las singulares características de la economía noruega, así como los hát tos culinarios de su población, hacían que la práctica totalidad de las importaci nes de aceite de oliva se dirigieran al sector conservero. Esto nos permitirá disp ner de un mercado único, tanto por su importancia, como por la ausencia de otr nichos de demanda que pudieran interferir en el análisis del objeto de estudio. $\mathrm{P}$ otro lado, la juventud de la industria conservera noruega, su desconocimiento d complejo sector del aceite de oliva, hizo que el suministro de este input queda en manos de los exportadores de los diferentes países productores. A lc conserveros noruegos, ante esta dependencia, sólo les quedó la posibilidad de cos trolar la calidad de los aceites importados y evitar los fraudes.

\section{EL ACEITE DE OLIVA EN LA INDUSTRIA CONSERVERA MODERN LAS CONSERVAS DE PESCADO EN NORUEGA EN EL PRIMER TERCIO DEL SIGLO XX}

Pese a que la posibilidad de conservar los alimentos mediante la esterilizacić se remonta a finales del siglo $\mathrm{XVIII}^{2}$, su difusión y expansión industrial no comer zaron hasta mediados del siglo $\mathrm{XIX}^{3}$. Fue la industria francesa la que tomó la in ciativa $^{4}$ y ejerció, en la práctica, su monopolio en el desarrollo de la modern conserva esterilizada del pescado. Sólo a partir de los años sesenta, nuevos pa ses, en primer lugar EE.UU. y España, iniciaron su andadura en este sector. A ellc se unieron, en la década de los ochenta, Portugal y Noruega. A este grupo de prir cipales países productores se incorporó Japón, a comienzos del siglo $\mathrm{XX}^{5}$.

A difereucia de los antiguos métodos de conservación del pescado, la modern industria conservera recurre a métodos más complejos, a la vez que exige la prє sencia de nuevos inputs que, en muchos casos, y debido a la dificultad para cor seguirlos, entorpecieron su crecimiento. Entre estos nuevos inputs se encuentra $\epsilon$ aceite de oliva ${ }^{6}$.

1. A comienzos de los años cuarenta, tras la Segunda Guerra Mundial, la industria conservera noruega er por su volumen de producción, la segunda del mundo, tras los EE.UU, y la primera respecto al volumen c exportación. La industria conservera, (1947), p. 10.

2. La invención de esta nueva técnica para conservar los alimentos se debe a Nicolás Appert, que cjerció c confitero en París en las úlimas décadas del siglo XVIII. A él se deben, asimismo, los primeros intentos para difnnd el nuevo procedimiento. En 1810 publicó Libre de tous les ou l'Art de Conserver pendant plusiers années tout les substances animales et vegetables. Citado por Abreu, F. (1983), p. 279.

3. Sobre los primeros pasos de esta invención, ver: Abreu, F. (1983), pp. 279-282, Carmona, X. (1985 pp. $177-178$.

4. Sobre la industria de conservas en Francia, ver: Carmona, X. (1985), D'Avigneu, A.M. (1958) y Decroiz J.L. (1964).

5. Carmona, X. (1994), pp. 128-129.

6. Carmona, X., (1994), p. 128. 
En el proceso de elaboración de las conservas de pescado, el aceite de oliva desempeña una función imprescindible y difícil de sustituir, al menos en las primeras décadas de vida de esta nueva industria. Son dos los momentos del proceso fabril en los que el aceite interviene: por un lado, en el freído, una vez que el pescado ha sido cortado, descabezado y esvicerado. En segundo lugar, tras el enlatado del producto, en el aceitado. Estos procedimientos requieren, obligatoriamente, aceites de oliva de gran calidad. De lo contrario el producto final dejaría mucho que desear, tanto en la presentación, como en el sabor, el olor, o, en el peor de los casos, en su deterioro total. Sólo cuando se logró perfeccionar el refino de los aceites de semillas se inició una seria amenaza para el aceite de oliva. La competencia se vio incrementada cuando, gracias a los avances en el proceso de refino y desodorización, el aceite de pescado pudo ser utilizado en estas funciones ${ }^{7}$.

En las décadas iniciales de la industria de conservas de pescado, el suministro de aceite a las fábricas que se fueron extendiendo por Europa era controlado por los franceses. El aceite de Niza era el más apreciado, el que se aseguraba para las conservas de mayor calidad y primeras marcas de las diferentes casas productoras. Su origen francés se resaltaba, situándolo en lugar bien visible de las latas ${ }^{8}$. En estos años el aceite español quedaba fuera de este mercado; su mala calidad, su deficiente refino, cuando lo era, le impedía el acceso a dicha industria. Ni siquiera en la naciente industria conservera española la proximidad de la producción de aceite, las abundantes cosechas o los bajos precios, propiciaron una penetración en el sector". Hubo que esperar a que, como consecuencia de la reacción ante la crisis agraria finisecular, se produjera una transformación radical de la industria aceitera ${ }^{10}$, lanzando al mercado aceites de calidad y a precios competitivos. En esta devolución, aunque los aceites catalanes ocuparon las primeras posiciones $^{11}$, los andaluces fueron paulatinamente desplazando al resto de los españoles ${ }^{12}$.

La industria conservera noruega es, por las circunstancias que se van a explicar, un caso paradigmático, a la vez que singular, de la progresión de la industria aceitera española en el mercado de la industria de conservas de pescado. El inicio de este sector en Noruega se remonta a finales de los años setenta y comienzos

7. En el caso noruego las consecnencias de esta ntilización del aceite de pescado, fundamentalmente de arenque, fueron nefastas para la exportación de aceite de oliva español. De 3.314 toneladas exportadas en 1935, se pasó a tan sólo 1.500, en 1952. Este proceso de snstitución se vio acelerado por las dificultades de la industria noruega para conseguir aceite de oliva en los añns de la posguerra española y la Segunda Guerra Mundial. La industria conservera (1947).

8. Abreu, F. (1998), pp. 9-I4, Gaya, M. (1930), p. 11.

9. Sobre la industria de conservas de pescado en España ver: Nadal, J. (1987), pp. 32-34, Carmona, X. (1983), Abreu, F. (1983), Barrón, J.I. (1992), Ríos, S. (1999).

10. Sobre la modernización de la industria aceitera: Jiménez, J.I. (1986), Zambrana, Feo. (1987), Parejo, A, y Zambrana, Fco. (1994), Hernández, S. (1997), Ramón, R. (1998).

11. Simpson, J. (1997), p. 286.

12. Carmona, X. (1994), p. 130. Sobre la evolución de la calidad de los aceites en las diferentes regiones productoras de España, ver: Zambrana, Fco. (1987), pp. 31 I-321. 
de los ochenta del siglo XIX, y se asentó, como en la mayor parte de las regione donde arraigó, sobre una tradicional e importante industria de salazón, fundamen talmente de bacalao y arenque, así como una importante flota ${ }^{13}$. Su progresión e: muy rápida, situándose en pocos años en el grupo de cabeza de países producto res, junto a Portugal, España, EE.UU. y Japón ${ }^{14}$. En el primer tercio del siglo XX los principales productos elaborados por esta industria como la gran riqueza de st plataforma costera. La abundancia de pesca en la costa oeste y sur del país y li posibilidad de realizar capturas a lo largo de todo el año ${ }^{15}$, dio lugar al estableci miento de un importante número de fábricas en distintas ciudades, entre las qu destaca la de Stavanger (cuadro 1).

El volumen de aceite de oliva importado por estos centros conserveros fluctuabi en función de las desiguales campañas de pesca, pero, como podemos observar eI el gráfico 1, la demanda no dejó de incrementarse, de tal modo que el mercadc Noruego se había convertido, para la industria aceitera española, en el octavo er importancia, durante el primer tercio del siglo XX (cuadro 2). Las ventas pasaror de 1.117 Tns., en 1906, a 3.314 Tns., en 1935.

En los años iniciales de esta industria, como ocurriera en otros países destaca dos en el sector, el suministro de aceite de oliva estuvo totalmente controlado po: los franceses ${ }^{16}$. El hecho de haber sido Francia el país impulsor de esta industric y la innegable realidad de que disponía, es estos años finales del siglo XIX y pri. meros del XX, de los aceites de mejor calidad, le garantizaba su hegemonía en estє mercado.

El predominio francés no se mantuvo durante mucho tiempo. Si observamo: el cuadro 3 comprobaremos que, desde fechas muy tempranas del siglo XX, la tendencia que se detecta es la de una paulatina pérdida de presencia de la indus. tria aceitera francesa a favor de la implantación de los otros países productores tendencia que se aceleró a partir de 1914, con una nítida preferencia por el aceite español.

Varios son los factores que explicarían esta consolidación del aceite español Por un lado, una circunstancia que resulta obvia, a la vista del cuadro 3: la Grar

13. Sobre la importancia de la flota en el crecimiento económico de Noruega, ver: Milward, A. y Saul, S.B (1979), pp. 494-497, Pollard, S. (1991), p. 280-281, Hildebrand, K.G. (1981), pp. 298-299.

14. La primera fábrica fue abierta por un cocinero francés en 1841: sin embargo, su expansión no se produjø hasta la década de los años ochenta. En 1879 había 4 fábricas, a mediados de los ochenta, 29 y, en 1920, cerca dı 200. Sobre la evolución de la industria de conservas de pescado en Noruega, ver: Pereira, E. (1943). Senner, F (19439, Hodne, F. (1975).

15. Al contrario de otros países, en los que la pesca se centraba en unos pocos meses, la riqueza de las costa: noruegas permitía un suministro regular a Io largo de todo el año. La clave estaba en la captura sucesiva de distinta: variedades de arenque. Durante el verano y el otoño se pescaba el "Brisling", que, una vez ahumado, se enlataba recubierto de aceite de oliva, y era vendido bajo el nombre de "sardinas noruegas. Brisling". En las misma: estaciones se capturaba el «Smaasild», un arenque pequeño, pariente del brisling. En primavera se pescaba el arenqut de gran tamaño. La caballa, el bacalao, la langosta, y otras especies eran trabajados también por la industri; conservera norucga. Ver: Senner, P. (1943), p. 5.

16. Carmona, X. (1985), p. I78, Gaya, M. (1930). 
CUADRO 1

PRINCIPALES CENTROS NORUEGOS IMPORTADORES DE ACEITE DE OLIVA EN 1926 (EN KGRS.)

\begin{tabular}{|c|c|c|c|c|c|c|}
\hline \multirow{3}{*}{ Ciudad } & \multicolumn{6}{|c|}{ País de Procedencia } \\
\hline & \multicolumn{2}{|c|}{ Francia } & \multicolumn{2}{|c|}{ Italia } & \multicolumn{2}{|c|}{ España } \\
\hline & Importación & $\%$ & Importación & $\%$ & Importación & $\%$ \\
\hline Fredrikshasld & 362 & 0,08 & 0 & 0,00 & 16.081 & 0,86 \\
\hline Fredrikstald & 22 & 0,01 & 0 & 0,00 & 9.468 & 0,50 \\
\hline Oslo & 34.744 & 8,11 & 2.347 & 0,52 & 0 & 0,00 \\
\hline Hamar & 9 & 0,00 & 0 & 0,00 & 0 & 0,00 \\
\hline Drammen & 200 & 0.05 & 0 & 0,00 & 0 & 0,00 \\
\hline Larvik & 0 & 0.00 & 0 & 0,00 & 20.205 & 1,08 \\
\hline Skicn & 98 & 0,02 & 0 & 0,00 & 0 & 0,00 \\
\hline Arendal & 12 & 0,00 & 0 & 0,00 & 0 & 0,00 \\
\hline Farsund & 2 & 0,00 & 0 & 0,00 & 0 & 0,00 \\
\hline Stavanger & 251.116 & 58,60 & 452.224 & 99,04 & 1.225 .727 & 65.27 \\
\hline Kopervik & 0 & 0,00 & 0 & 0,00 & 34.739 & 1.85 \\
\hline Haugesund & 8 & 0,00 & 0 & 0,00 & $62.86 \mathrm{l}$ & 3.35 \\
\hline Odda & 1.000 & 0,23 & 0 & 0,00 & 0 & 0,00 \\
\hline Bergen & 17.666 & 4,12 & 22 & 0,00 & 262.914 & 14,00 \\
\hline Floro & 2.743 & 0,64 & 0 & 0,00 & 14.108 & 0,75 \\
\hline Aslesund & 8.901 & 2,08 & 0 & 0,00 & 34.035 & 1,81 \\
\hline Molde & 0 & 0,00 & 0 & 0,00 & 1.017 & 0,05 \\
\hline Kristisusund & 5.596 & 1,31 & 3 & 0,00 & 5.583 & 0,30 \\
\hline Trondjom & 105.944 & 24.73 & 2.015 & 0,44 & 161.017 & 8,57 \\
\hline Namso & 3 & 0,00 & 0 & 0,00 & 0 & 0,00 \\
\hline Bods & 0 & 0,00 & 0 & 0,00 & 23.957 & 1,27 \\
\hline Harstad & 0 & 0,00 & 0 & 0,00 & 6.361 & 0,34 \\
\hline Vardo & 100 & 0,02 & 0 & 0,00 & 0 & 0,00 \\
\hline Total & 428.526 & 100,00 & 456.611 & 100,00 & 1.878 .073 & 100,00 \\
\hline
\end{tabular}

Fuente: F.E.A.O.E. Biblioteca de la Facultad de CC.EE. y EE. Unv, Málaga ${ }^{17}$.

17. Entre las fuentes utilizadas para claborar este trabajo ocupa un lugar muy destacado los fondos documentales de la Federación de Exportadores de Aceite de Oliva de España, en adelante: F.E.A.O.E. Estos fondos se ubican en la Biblioteca de la Facultad de CC.EE. y EE. de la Universidad de Málaga. Agradezco al profesor Juan Francisco Zambrana que me haya facilitado su localización. 
CUADRO 2

EXPORTACIÓN ESPAÑOLA DE ACEITE DE OLIVA A LOS PRINCIPALES MERCADOS, 1900-1930 (EN QMS.). MEDIA ARITMÉTICA POR PERÍODOS

\begin{tabular}{lrrrrrr}
\hline & \multicolumn{2}{c}{ Período $1901-1903$} & Período $1911-1913$ & \multicolumn{2}{c}{ Período 1926-1930 } \\
\cline { 2 - 7 } Países & Exportación & $\%$ & Exportación & $\%$ & Exportación & $\%$ \\
\hline EE.UU. & 7.509 & 1,76 & 15.065 & 3,55 & 130.096 & 15,89 \\
Argentina & 8.099 & 1,90 & 77.354 & 18,24 & 131.537 & 16,06 \\
Cuba & 39.889 & 9,35 & 42.639 & 10,05 & 65.794 & 8,03 \\
Uruguay & 2.733 & 0,64 & 3.667 & 0,86 & 69.596 & 8,50 \\
Alemania & 20.100 & 4,71 & 2.776 & 0,65 & 4.870 & 0,59 \\
Francia & 62.630 & 14,67 & 51.686 & 12,19 & 29.588 & 3,61 \\
Italia & 102.667 & 24,05 & 106.062 & 25,01 & 197.911 & 24,17 \\
Gran Bretaña & 87.780 & 20,57 & 27.709 & 6,53 & 29.013 & 3,54 \\
Noruega & 3.274 & 0,77 & 5.604 & 1,32 & 19.275 & 2,35 \\
Otros países & 92.157 & 21,59 & 91.572 & 21,59 & 141.264 & 17,25 \\
Total & $\mathbf{4 2 6 . 8 3 8}$ & $\mathbf{1 0 0 , 0 0}$ & $\mathbf{4 2 4 . 1 3 4}$ & $\mathbf{1 0 0 , 0 0}$ & $\mathbf{8 1 8 . 9 4 4}$ & $\mathbf{1 0 0 , 0 0}$ \\
\hline
\end{tabular}

Fuente: F.E.A.O.E., Biblioteca de la Facultad de CC.EE. y EE. Univ. Málaga.

Guerra Europea. Este conflicto involucró a Francia e Italia, y entregó el mercado conservero noruego en manos de los exportadores españoles. Sin embargo, esta es una circunstancia coyuntural, que afectó a otros sectores de la economía española, incluso a otros mercados de la propia industria aceitera, y sus consecuencias positivas no fueron mucho más allá de unos excepcionales años de beneficios especulativos ${ }^{18}$. No parece que fuera este el caso del mercado noruego. Quiere esto decir que, para explicar la sólida hegemonía conseguida por el aceite español en este mercado, tendremos que recurrir a otros factores distintos a los meramente coyunturales.

Desde nuestro punto de vista, para entender esta progresión es necesario, antes de nada, resaltar la singularidad del mercado noruego. A diferencia de otros, en los que la demanda de aceite tenía su origen en un variado abanico de necesidades ${ }^{19}$, en el caso noruego la demanda partía, exclusivamente, del sector de conservas de pecado ${ }^{20}$. Un mercado nuevo, en el que el peso del componente cultural

18. Sobre las consecuencias de la Guerra Europea en las exporlaciones españolas de aceite ver: Zambrana, Fco. (1987), pp. 305-306; Hernández, S. (1997), pp. 247-253; Simpson, J. (1997), p. 287.

19. No es fácil simplificar la demanda de aceite en estos años finales del siglo XIX y primeros del XX. A una demanda industrial con destino diverso y en claro retroceso (jabones, cosmética, lubricantes, pinturas, combustible para la iluminación, etc.), hay que sumar el ascendente mercado del «aceite de boca», a su vez de gran heterogeneidad, el de la industria conservera, en sus diferentes ramas, u otros de menor entidad,

20. La importación đe aceite de oliva para otros usos es insignificante. Es más, aunque desde los años veinte la asociación de conserveros noruegos emprendió interesantes estudios con el objetivo de diferenciar que tipo de 
CUADRO 3

IMPORTACIÓN DE ACEITE DE OLIVA EN NORUEGA, 1900-1936 (EN TNS.)

\begin{tabular}{|c|c|c|c|c|c|c|c|c|c|}
\hline Año & Total & Francia & $\begin{array}{c}\% \text { del } \\
\text { total }\end{array}$ & España & $\begin{array}{c}\% \text { del } \\
\text { total }\end{array}$ & Italia & $\begin{array}{c}\% \text { del } \\
\text { total }\end{array}$ & Otros & $\begin{array}{c}\% \text { del } \\
\text { total }\end{array}$ \\
\hline 1906 & 1.117 & 1.074 & 96,15 & 36 & 3,22 & 7 & 0,63 & - & 0,00 \\
\hline 1907 & 807 & 713 & 88,35 & 22 & 2,73 & 72 & 8,92 & - & 0,00 \\
\hline 1908 & 1.104 & 715 & 64,76 & 101 & 9,15 & 288 & 26,09 & - & 0,00 \\
\hline 1909 & 1.355 & - & - & - & - & - & - & - & - \\
\hline 1910 & 1.355 & - & - & - & - & - & - & - & - \\
\hline 1911 & 1.606 & 805 & 50,12 & 460 & 28,64 & 341 & 21,23 & - & 0,00 \\
\hline 1912 & 1.321 & - & - & - & - & - & - & - & - \\
\hline 1913 & 1.035 & 459 & 44,35 & 258 & 24,93 & 318 & 30,72 & - & 0,00 \\
\hline 1914 & 2.428 & 906 & 37,31 & 1.035 & 42,63 & 205 & 8,44 & 282 & 11,61 \\
\hline 1915 & 5.779 & 676 & 11,70 & 4.904 & 84,86 & 100 & 1,73 & 99 & 1,71 \\
\hline 1916 & 3.353 & 109 & 3,25 & 3.212 & 95,79 & 22 & 0,66 & 10 & 0,30 \\
\hline 1917 & 1.759 & 32 & 1,82 & 1.669 & 94,88 & 0 & 0,00 & 58 & 3,30 \\
\hline 1918 & 737 & 0 & 0,00 & 737 & 100,00 & 0 & 0,00 & 0 & 0,00 \\
\hline 1919 & 1.456 & 38 & 2,61 & 1.018 & 69,92 & 0 & 0,00 & 400 & 27,47 \\
\hline 1920 & 1.101 & 459 & 41,69 & 514 & 46,68 & 22 & 2,00 & 106 & 9,63 \\
\hline 1921 & 484 & 37 & 7,64 & 325 & 67,15 & 27 & 5,58 & 95 & 19,63 \\
\hline 1922 & 2.003 & 193 & 9,64 & 1.766 & 88,17 & 29 & 1,45 & 15 & 0,75 \\
\hline 1923 & 1.909 & 319 & 16,71 & 1.542 & 80,78 & 29 & 1,52 & 19 & 1,00 \\
\hline 1924 & 4.489 & 447 & 9,96 & 3.099 & 69,04 & 103 & 2,29 & 840 & 18,71 \\
\hline 1925 & 2.141 & 266 & 12,42 & 1.750 & 81,74 & 0 & 0,00 & 125 & 5,84 \\
\hline 1926 & 2.788 & 429 & 15,39 & 1.880 & 67,43 & 457 & 16,39 & 22 & 0,79 \\
\hline 1927 & 3.178 & 566 & 17,81 & 2.405 & 75,68 & 73 & 2,30 & 134 & 4,22 \\
\hline 1928 & 3.249 & 497 & 15,30 & 2.715 & 83,56 & 17 & 0,52 & 20 & 0,62 \\
\hline 1929 & 4.741 & 824 & 17,38 & 2.399 & 50,60 & 849 & 17,91 & 669 & 14,11 \\
\hline 1930 & 2.668 & 153 & 5,73 & 2.387 & 89,47 & 7 & 0,26 & 121 & 4,54 \\
\hline 1931 & 1.342 & 56 & 4,17 & 1.240 & 92,40 & 14 & 1,04 & - & 0,00 \\
\hline 1932 & 3.856 & - & 0,00 & 3.035 & 78,71 & - & 0,00 & - & 0,00 \\
\hline 1933 & 3.222 & 295 & 9,16 & 2.375 & 73,71 & 250 & 7,76 & 302 & 9,37 \\
\hline 1934 & 2.228 & 86 & 3,86 & 2.013 & 90,35 & 5 & 0,22 & 125 & 5,57 \\
\hline 1935 & 3.314 & - & 0,00 & - & 0,00 & - & 0,00 & 3.314 & 100,00 \\
\hline 1936 & - & - & - & - & - & - & - & - & - \\
\hline
\end{tabular}

Fuente: 1906-1932, Tiddsskrift for Hermetikindustri (recogidos por la F.E.A.O.E., Informe 1.833, a partir de 1933, otras fuentes. 
o histórico, que tan presente estuviera en otros mercados aceiteros ${ }^{21}$, está ausente Asimismo, la variable proximidad geográfica ${ }^{22}$, que es otra de las que explican e incremento del comercio bilateral, tampoco tendría, en nuestro caso, mayor peso La distancia que separa a España de Noruega no se diferencia mucho de la existente entre este país y los demás grandes centros exportadores de aceite de oliva Sólo en el hecho de que era España la que suministraba la sal a la tradicional industria noruega de salazón, podemos encontrar un precedente al suministro de aceite $^{23}$.

A nuestro modo de ver habría que considerar como elemento de mayor peso la rigidez de la demanda noruega de aceite de oliva. Es un aceite que se utiliza en la industria conservera, exclusivamente. Es cierto que otros aceites de oliva habrían podido satisfacer esa demanda, incluso otros aceites vegetales. Sin embargo, las exigencias de calidad de la industria noruega de conservas de pescado ${ }^{24}$ y la necesidad de mantener una homogeneidad y continuidad en el suministro de este input, haría que las condiciones para consolidarse en el mercado quedaran en el lado de la oferta. A mantener la hegemonía, en un escenario sin grandes cambios. contribuiría la confianza en lo que es conocido.

La clave está en cómo el aceite alcanzó a cumplir las condiciones que le imponía el mercado noruego de la industria conservera. Si el aceite español no hubiera logrado unos elevados niveles de competitividad y de adecuación a la demanda, no cabe la menor duda que, una vez superado el conflicto bélico, el mercado noruego se hubiera perdido, dada la homogeneidad de su demanda, en su totalidad. Sin embargo, tras una débil recuperación por parte del aceite francés, la industria conservera noruega se decanta, de forma clara y progresiva, por el aceite de origen español.

accites de oliva sería más apropiado para las distintas conservas de pescado, en la década de los años treinta aún no habían llegado a conclusiones significativas y seguían inclinándose por la importación de un accite cstándar, cuyas bondades les eran conocidas. Para las investigaciones de la industria conservera noruega sobre la idoneidad de los diferentes tipos de aceites, ver los trabajos de Lunde, G. (19.33), Lunde, G. y Mathiesen, E. (19.32), Lunde, G., Mathiesen, E. y Mikkelsen, E. (1933), Mathiesen, E. (1932), en la revista Tidsskrift for Hermetikindustr, Años 1932, núm. 18 y 1933, núm. 19, entre otros.

21. Desde el punto de vista tcórico la influencia del factor cultural e histórico, en gran medida relacionado a los movimientos migratorios, ver: Gould (1994, Eichengreen e Irwin (1996).

22. Linneman (1996).

23. España y Noruega mantenían unas relaciones comerciales en las que la exportación de productos agrícolas -agrios, frutos secos, vinos y licores-, además del aceite de oliva, ocupaban, junto al plomo, los pritneros lugares. En la importación española de productos noruegos ocupaban los lugares más destacados los productos relacionados con la industria pesquera, bacalao y esperma de ballena, fundamentalmente, y de la industria maderera, fundamentalmente pasta de papeI. Ver: Jacobsen, N. (1953).

24. Las importaciones medidas para garantizar la calidad de los productos de esta industria convirtieron a Noruega en uno de los paises punteros en la investigación de los inputs utilizados, así como en los procesos técnicos para la elaboración de estas conservas. En estos objetivos se produjo una coincidencia entre las autoridades noruegas y el empresariado del sector. El resultado fue la creación de un instituto específico, órgano semioficial, cuyo consejo era nombrado por el munarca, en el que destacan los estudios realizados por el "Hermetikindustriens laboratoritum". Una descripción sintética de los avances conseguidos por esta industria se pnede encontrar en: Hann, W.S. (1948). 
¿Cuáles son las claves de ese éxito? En primer lugar, la calidad. El aceite español, especialmente el catalán, había logrado en los años finales del siglo XIX y, sobre todo, en el primer tercio del XX, unos niveles de calidad superiores, en muchos casos, a la de los mejores aceites franceses o italianos ${ }^{25}$. Además, a una industria como la noruega de conservas de pescado, fuertemente preocupada por la calidad de los inputs, la gran producción de la industria aceitera española le proporcionaba confianza en cuanto a la ausencia de mezclas nefastas. Garantía que no estaba asegurada en industrias deficitarias, como la francesa e italiana, basadas en la mezcla de aceites de muy divergo origen ${ }^{26}$.

El reconocimiento a la calidad del aceite español y su adecuación a las necesidades de la industria conservera noruega fue reiteradamente manifestado, no sólo por las instancias científicas de este país ${ }^{27}$, sino por otros países productores de aceite $^{2 x}$. Esta industria era especialmente cuidadosa a la hora de seleccionar Ios aceites de oliva que deberían usarse en las conservas de pescado. Al contrario de lo que ocurría en España o Portugal, donde no sólo se usaban mezclas en las que el aceite refinado de oliva suponía el mayor porcentaje o la totalidad, sino que, en el caso portugués, se recurre a otros aceites vegetales, los aceites importados por Noruega deberían tener un muy elevado porcentaje de aceite virgen, al que se le asignaba un mayor poder de conservación, a la vez que un bajo nivel de ácido libre ${ }^{29}$.

De los estudios desarrollados en Noruega por el Hermetikkindustriens laboratorium, entre 1929 y 1933, se desprenden diferencias importantes en las características de los aceites importados de diferentes países (cuadro 4 y 5). Dadas las exigencias de este mercado, los aceites franceses tenían pocas posibilidades. Su elevado índice de enranciamiento $(7,39) \mathrm{y}$, sobre todo, su alto contenido de aceites refinados (índice de fluorescencia de 149,86), los colocaba por debajo de los aceites españoles. En similares circunstancias se encontrarían los aceites de

25. Sobre la modernización del olivar y la industria aceitera española ver Zambrana, Fco. (1987).

26. Sobre la importancia de conocer el origen de los aceites y la desconfianza de los conserveros noruegos respecto al origen de algunos aceites, ver: Lnnde, G. (1933), pp. 112-117 y Lunde, G., Mathiesen, E., Mikkelsen, E. (1933), 375-382.

27. En lo referente a la calidad de los aceites importados por Noruega, en su inmensa mayoría procedentes de España, las investigaciones anteriormente citadas eran concluyentes: «...No hemos podido, en ningún caso indic'ar la presencia de aceites extraños en el aceite de oliva (...). Nosotros tenemos la impresión cierta de que la calidad del aceite de oliva importados en estos últimos años es más igual.... La preocupación de la industria conservera noruega por la calidad y por el control de las mezclas, les llevó a desarrollar una tecnología específica destinada a detectar los fraudes, adulteraciones y tipo de mezclas que caracterizaban a este producto. Esta tecnología se desarrolla en: Lunde, G. (1933), pp. 112-117 y Lunde, G., Mathiesen, E., Mikkelsen, E. (1933), 375-382.

28. En referencia a la confianza que los conserveros noruegos tenían en cl aceite español, la prensa especializada italiana, L'Olivicultore, manifestaba en 1929, año de precios altos en el accite español, "...En los veinte años áltimos se considera en Noruega que el aceite de oliva español es el más indicado para la conservación del "Sprat" y de los "arenques pequeños"...". L'Olivicultore, 25 de mayo de 1929, p. 2.

29. La legislación noruega sobre la industria de conservas de pescado exigía que el aceite de oliva no pudiera tener más de un $30 \%$ de aceite refinado. Asimismo, la cantidad de ácido libre, acidez, no debería sobrepasar el $2 \%$. Lunde, G., Mathiesen, E., y Mikkeisen, E. (1933). 
CUADRO 4

CALIDAD DEL ACEITE DE OLIVA DE LOS PRINCIPALES PAÍSES

PRODUCTORES (CAMPAÑA 1932-1933) ${ }^{30}$

\begin{tabular}{lccccccc}
\hline Procedencia & ID & IR & IF & PC & IE & AL & IY \\
\hline Cataluña & 0,92 & 1,47 & 110,48 & 1,13 & 6,00 & 1,12 & 83,27 \\
Andalucía & 0,92 & 1,47 & 147,00 & 1,00 & 4,59 & 1,08 & 82,87 \\
Madrid & 0,92 & 1,47 & 294,00 & 1,50 & 3,30 & 0,63 & 83,00 \\
España & 0,92 & 1,47 & 130,50 & 1,08 & 5,34 & 1,09 & 83,09 \\
Francia & 0,92 & 1,47 & 149,86 & 1,58 & 7,39 & 0,77 & 84,15 \\
Italia & 0,92 & 1,47 & 252,00 & 2,67 & 6,22 & 1,06 & 83,02 \\
Túnez & 0,92 & 1,48 & 102,00 & 1,00 & 11,75 & 1,02 & 89,10 \\
Levante & 0,92 & 1,47 & 170,00 & 1,00 & 9,49 & 1,91 & 84,47 \\
\hline
\end{tabular}

Fuente: Lunde, G., Mathiesen, E. y Mikkelse, E. (1933),

ID: Índice de densidad, IR: Índice de refracción, IF: Índice de fluorescencia, PC: Punto de congelación

IE: Índice de enranciamiento, AL: Ácido libre (acidez), IY: Índice de yodo.

\section{CUADRO 5}

CALIDAD DEL ACEITE DE OLIVA DE LOS PRINCIPALES PAÍSES

PRODUCTORES (CAMPAÑA 1929-1933)

Densidad a $15^{\circ} \mathrm{C} \quad$ Índice de refracción Glíndice de Yodo Gliceridos

Origen Campaña Máxima Mínima Media Máximo Minimo Medio Máximo Mínimo Medio Máx. Mín. Med.

$\begin{array}{llllllllllllll}\text { España } & 1929-1930 & 0,9170 & 0,916 & 0,917 & 1,4677 & 1,4672 & 1,467 & 85,5 & 83,0 & 84,5 & 1 & 5 & 2 \\ & 1930-1931 & 0,9171 & 0,916 & 0,917 & 1,4677 & 1,4670 & 1,467 & 86,3 & 82,4 & 83,7 & 1 & 5 & 2 \\ & 1931-1932 & 0,9172 & 0,916 & 0,916 & 1,4674 & 1,4668 & 1,467 & 86,8 & 79,9 & 83,1 & 1 & 4 & 2 \\ & 1932-1933 & 0,9166 & 0,916 & 0,916 & 1,4672 & 1,4668 & 1,467 & 86,2 & 79,4 & 83,3 & 1 & 2 & 1\end{array}$

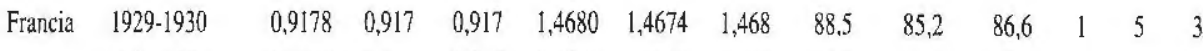

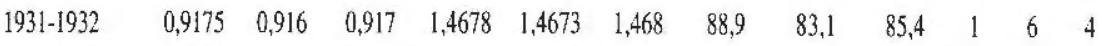

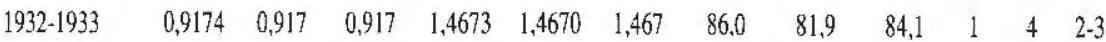

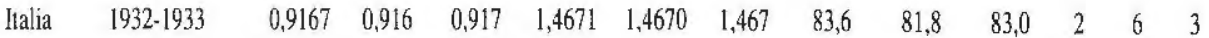

Fuente: Lunde, G., Mathiesen, E. y Mikkelse, E. (1933).

30. La fuente facilita una inayor desagregación, diferenciando tipo de envases y ciudad de procedencia de las diferentes partidas, que, por razones de espacio han sido omitidas. 
Italia - con un muy elevado índice de fluorescencia y de enranciamiento- o los de Túnez y Esmirna. No cabe duda que los mejor posicionados eran los aceites españoles y, singularmente, los catalanes. Sus características -sabor suave, reducido índice de enranciamiento y baja acidez- les permitían obtener aceites apropiados a la demanda de la industria conservera noruega sin tener que abusar de la mezcla de aceite refinado (índice de fluorescencia del 110,48).

Estas circunstancias, unido al importante volumen de la cosecha española y a los tradicionales y sólidos conocimientos del sector, hacía que los industriales y exportadores españoles, y singularmente los catalanes, pudieran ofrecer un tipo de aceite estándar, con una elevada homogeneidad en las diferentes campañas. Unas condiciones que, como podemos observar en el cuadro 5, otros países tenían mayor dificultad en satisfacer.

En segundo lugar, el precio. Difícilmente podríamos explicar el incremento de las exportaciones a Noruega con el simple recurso a la favorable coyuntura bélica o a la calidad de los aceites españoles. No cabe duda que, descartados los elementos culturales o la proximidad geográfica, la cotización en el mercado internacional del aceite español y su relación con sus más próximos competidores ha de ser un factor decisivo para comprender este éxito.

Aunque el precio del aceite de oliva en el mercado internacional está íntimamente ligado a la evolución del precio de otras grasas vegetales ${ }^{31}$, en el mercado noruego la relación habría que establecerla con las cotizaciones del aceite de oliva de otras procedencias, fundamentalmente francesa e italiana ${ }^{32}$. En la evolución seguida por los precios de este producto, a lo largo del período que nos interesa, podemos diferenciar varias etapas. En una primera, anterior al desarrollo de la industria conservera noruega, los precios más competitivos en el mercado internacional correspondieron al aceite italiano. Sin embargo, a partir de $1880 \mathrm{y}$, sobre todo, a partir de 1890 , con la devaluación de la moneda española, la cotización del aceite español logró unos precios más competitivos que los aceites italianos y franceses. La ventaja definitiva sino la consolidación y expansión, tras la crisis agraria finisecular, del olivar en España y su retroceso en Francia e Italia.

Como podemos observar en el cuadro 6, Ios precios del aceite español permanecieron siempre por debajo de las cotizaciones del aceite italiano, en el período de 1927-1934. Sólo los aceites de Túnez, importados en grandes cantidades por Francia e 1talia para elaborar sus mezclas, y los aceites de Levante podrían competir con los de España. Sin embargo, como ya hemos visto, estos aceites no ofrecían la calidad requerida por el mercado noruego.

31. Sobre la evolución del mercado internacional de las grasas vegetales, ver: Zambrana, Fco. (1984).

32. Sólo en 1929, año en el que los precios del aceite español cotizó, durante unos meses, a unos precios superiores a los de sus competidores, los conserveros noruegos imporaron aceite de Esmima. Fue una importación de escaso volumen y poco satisfactoria para la industria noruega (F.E.A.O.E., 1929. Informe 723). 
EVOLUCIÓN DEL PRECIO DE ACEITE DE OLIVA EN LOS PIRINCIPALES PAÍSES

\begin{tabular}{lcccccc}
\hline & \multicolumn{2}{c}{ España (Tortosa fino) } & \multicolumn{2}{c}{ Italia (Bari soprafino) } & \multicolumn{2}{c}{ Túnez (1 $1^{\text {a }}$ presión) } \\
\cline { 2 - 6 } Año & Pesetas & Francos oro & Liras & Francos oro & Francos & Francos oro \\
\hline 1927 & 262 & 232 & 953 & 252 & 1.128 & 229 \\
1928 & 217 & 186 & 802 & 219 & 905 & 183 \\
1929 & 222 & 170 & 636 & 173 & 720 & 146 \\
1930 & 181 & 109 & 494 & 135 & 525 & 107 \\
1931 & 211 & 104 & 554 & 151 & 558 & 113 \\
1932 & 206 & 86 & 471 & 125 & 402 & 82 \\
1933 & 171 & 74 & 397 & 107 & 311 & 52 \\
1934 & 171 & 74 & 484 & 129 & 334 & 68 \\
\hline
\end{tabular}

Fuente: L'Olivicultore, núm. 3, marzo de 1935. p. 37.

En tercer lugar, la competencia de los empresarios exportadores y lo acertadc de sus estrategias en este mercado. Es cierto, como indica R. Ramón ${ }^{33}$, que los empresarios franceses e italianos dispusieron de mejores condiciones de partida Su experiencia industrial y comercial, sus conocimientos del mercado, así comc un marco institucional muy favorable, les proporcionaron unas condiciones $\mathrm{d} \epsilon$ partida difíciles de superar. Esto explicaría su dominio del mercado en el últimc tercio del siglo XIX y primeros lustros del XX. Sin embargo, la industria aceiter española no estaba huérfana de posibilidades. Su mayor potencial productivo y sus menores costes de producción tal vez fueran las bazas más importantes, pero er absoluto hemos de desdeñar el esfuerzo realizado por el empresariado del sector para superar las barreras de entrada que les imponía el mercado exterior. Comc sabemos por los estudios de Zambrana, Parejo, Jiménez, Zapata y Hemández, la competitividad del aceite español ganó muchos enteros en el primer tercio del siglo XX. No sólo se produjo la modernización del cultivo y del proceso de fabricación, facetas que repercutieron directamente en la mejora de la calidad, sino que. parejo a estos cambios, se desarrolló una activa penetración del empresariado español en el mercado exterior.

El caso del mercado noruego es más que significativo. Una vez que dispusieron de un producto de calidad, en cantidad suficiente y a precios competitivos, las estrategias seguidas por los exportadores españoles fueron mucho más agresivas que las de los empresarios italianos o franceses. A este respecto, cabe destacar: en primer lugar, el ofrecimiento de un aceite de calidad y adaptado al gusto de los

33. Ramón. R. (1997), pp. 49-53. 
conserveros noruegos ${ }^{34}$. El aceite que llegaba a Noruega era obtenido por el procedimiento de mezclas de diferentes aceites de oliva, refinado y virgen ("coupages»), en las que el tipo "Tortosa» tenía un lugar preferente ${ }^{35}$. Cada año estas mezclas alcanzaba una mayor homogeneidad ${ }^{36}$, consiguiendo un aceite caracterizado por su gran brillantez, una acidez inferior a un grado y un buen paladar. En segundo lugar, el cuidado puesto por los exportadores españoles en que el producto fuera de la mayor calidad y no sufriera alteraciones por su almacenaje o transporte. El aceite que se enviaba a Noruega era embalado en barriles de madera de castaño que, a diferencia de los de roble, evitaba la alteración en el sabor del aceite. En los años treinta estos envases estaban siendo sustituidos por otros de acero, interiormente estañados. En tercer lugar, el seguimiento del mercado y las ventajosas formas de pago. Los exportadores españoles habían conseguido una sólida red de agentes de venta en Noruega, con su centro en Stavanger, a la vez que visitaban regularmente a sus compradores, con objeto de tener un conocimiento puntual de sus preferencias. Asimismo, tenían establecidas formas de pago, entre 30 y 60 días, más ventajosas que la de sus competidores.

\section{EMPRESAS Y EMPRESARIOS ESPAÑOLES EN EL MERCADO NORUEGO}

El análisis de las empresas presentes en el mercado de la industria noruega de conservas de pescado se puede hacer desde diversas perspectivas. La búsqueda de elementos que permitan establecer diferencias entre ellas debe de ser una. Nuestra atención ha de estar puesta, asimismo, en la posibilidad de establecer niveles de especialización entre las diferentes regiones españolas productoras de aceite. Un tercer enfoque ha de ser contrastar la presencia e importancia de la actividad de estas empresas en el mercado noruego, del que se ha destacado ya su singularidad, con respecto a los mercados más importantes del aceite español.

Las fuentes para este análisis son las declaraciones juradas presentadas por las empresas exportadoras españolas para el período de tiempo de $1930-1935^{37}$, el

34. Los propios competidores italianos reconocían esta calidad. El órgano de prensa L'Olivicultore, refiriéndose, en 1929 a los mayores precios que en esos meses tenía el accite español indicaba: «En el último número de la revista de' los fabricantes noruegos de conservas alimenticias, éstos hacen interesantes apreciaciones dignas de conocerse, declarándose, desde luego, decididos protectores del mercado español. Se afirma rotundamente en dicho órgano de publicidad, que el aceite de oliva español es de superior calidad al de otras procedencias, y que en cuanto a la aplicacion de la industria conservera reúne cualidades intrinsecas inmejorubles. haciendo sobre salir la excelencia del aceite español...*. Recogido por la Hoja Informativa, F.E.A.O.E., Informe 723, año 1929.

35. Gaya, M. (1430), p. I1.

36. En los repetidos análisis que los laboratorios de la industria conservera noruega realizó de los acettes importados, así como en las constataciones que el químico Mathiessen lleva a cabo en nuestro país, se pone de manifiesto este procedimiento y sus buenos resultados.

37. Estas declaraciones, en Ias que figuran las exporaciones a la totalidad de los mercados aceiteros llevadas a cabo por las empresas exportadoras españolas, fueron recogidas por la FEAOE, en octubre de 1935. Sin embargo, 
Registro Oficial de Exportadores, creado por R.O. de 11 de diciembre de $1929^{3 \varepsilon}$ los Libros de Registro de Sociedades del Registro Mercantil de las diferentes pro vincias andaluzas y los Anuarios Financieros. Desgraciadamente estas fuentes nı nos permiten establecer un seguimiento de la implantación de las diferentes em presas a lo largo del período estudiado, pues los datos de los que disponemo sólo hacen referencia al quinquenio de 1930-1935. Habrá que esperar a que sı lleven a cabo estudios monográficos sobre estas sociedades para comprende como fueron sus trayectorias en este y otros mercados. Mientras tanto, alguna conclusiones sí que podremos obtener, por pobres que estas sean, de los dato disponibles.

En primer lugar, se constata que, pese a las singularidades del mercado norue go, no son pocas las empresas exportadoras que están presentes en él (cuadro 7) De las 49 recogidas en el apéndice 1, veintiuna mantienen relaciones comerciale: con la industria conservera de este país. Esto nos indicaría que, dadas las exigen cias de calidad y el tipo de aceite demandado por la industria conservera noruega existía un empresariado activo, conocedor del mercado exterior y dispuesto a lu char por penetrar en él.

En segundo lugar, una agrupación de estas empresas por su origen, pone dt manifiesto el claro predominio del sector exportador catalán sobre la otra gran áre productora, Andalucía. Nada de extraño tiene este mayor peso de la industria acei. tera catalana. Como hemos indicado, esta industria fue la primera en consegui] aceites de calidad y desarrollar el sector del refino ${ }^{39}$. Asímismo, los aceites cata. lanes se adaptaban singularmente a las exigencias de los conserveros noruegos ${ }^{40}$ Sus aceites vírgenes tenían un sabor más suave, menos intenso que los andaluces por lo que no requerían ser rebajados con aceite refinado. Del mismo modo, sı experiencia comercial y su implantación en los mercados exteriores eran muy superiores al resto de las regiones productoras españolas ${ }^{41}$.

Más llamativa es la importante presencia de empresas andaluzas en este mercado. De las 21 empresas que exportaban a Noruega, 10 estaban radicadas er Andalucía. Su implantación en este mercado sería más reciente, según se deduce de la fecha de constitución de la mayor parte de ellas. Como se verá más adelan. te, estas empresas, por su objeto social, por el volumen de capital que movilizan

no todas las empresas exportadoras españolas habrían entregado estas declaraciones. El Registro de Exportadores llega a contabilizar hasta 80 empresas (Gaceta de Madrid, 11 de enero de 1933). Ignoramos la importancia de las ausentes en el volumen total del aceite exportado y sus mercados preferentes. No obstante, entendemos que las que entregaron la documentación exigida a la F.E.A.O.E. serían las más importantes del sector.

38. Gaceta de Madrid del 29 de diciembre de 1929. En 1932, el gobierno de la República, por Orden del \& de octubre de 1932, del ministerio de Agricultura, Industria y Comercio, confirmó este Registro de Exportadores

39. Simpson, J. (1997), p. 286.

40. Como se ha indicado en otro Iugar, la industria conservera noruega exigía que el aceite utilizado fuera, a] menos, en un $70 \%$ virgen. Los aceites andaluces vírgenes tenían un fuerte sabor al fruto, por lo que dado los gustos de la demanda, se solían mezclar en una proporción del 30\% virgen y $70 \%$ refinado.

41. Sobre la temprana presencia de los exportadores catalanes en los distintos mercados, ver Ramón, R. (1997). 


\section{CUADRO 7}

EXPORTACIÓN DE ACEITE DE OLIVA ESPAÑOL A NORUEGA, 1930-1935. EXPORTADORES Y ORIGEN DE LA EXPORTACIÓN

\begin{tabular}{|c|c|c|c|c|c|c|c|}
\hline Empresa & 1 & 2 & 3 & 4 & 5 & 6 & 7 \\
\hline Carbonell (Córdoba), S.C. & Córdoba & 1866 & 1.243 .955 & 9,61 & 31.726 .331 & 3,92 & 0,61 \\
\hline Dulken (E. Van), R.C. & Málaga & 1932 & 392.401 & 3,03 & 12.069 .936 & 3,25 & 0,19 \\
\hline Luca de Tena (Hijos de), R. & Sevilla & 1891 & 4.850 & 0,04 & 6.205 .656 & 0,08 & 0,00 \\
\hline Maestroni, S.A. & Málaga & 1926 & 1.328 .900 & 10,27 & 6.783 .824 & 19,59 & 0,65 \\
\hline Minerva, S.A. & Málaga & 1919 & 337.255 & 2,61 & 29.523 .257 & 1,14 & 0,16 \\
\hline Pallarés Hernanos, R.C. & Córdoba (Cabra) & 1917 & 708.278 & 5,47 & 8.085 .614 & 8,76 & 0,34 \\
\hline Riva Hermanos, S.A. & Málaga y Jaén & 1926 & 75.974 & 0,59 & 6.410 .562 & 1,19 & 0,04 \\
\hline Utrerana, S.A, La & Sevilla (Utrera) & 1923 & 15.103 & 0,12 & 205.040 & 7,37 & 0,01 \\
\hline Ibarra (Hijos de), S.C. & Sevilla & 1885 & 61.300 & 0,47 & 27.776 .036 & 0,22 & 0,03 \\
\hline Moro, S.A. & Barcelona & 1926 & 321.800 & 2,49 & 19.589 .900 & 1,64 & 0,16 \\
\hline Total Andalucia & - & - & 4.489 .816 & 34,69 & 148.376 .156 & 3,03 & 2,19 \\
\hline Ballester Romero, Juan & Tarragona (Tortosa) & - & 2.798 .638 & 21,62 & 8.948 .011 & 31,28 & 1,36 \\
\hline Basseda, Antonia & Barcelona & 1850 & 758.108 & 5,86 & 1.047 .862 & 72,35 & 0,37 \\
\hline Fontana, Enrique & Tamagona & - & 112.518 & 0,87 & 6.647 .874 & 1,69 & 0,05 \\
\hline Gasull (Esl. Félix) & Tarragona (Reus) & 1922 & 898.156 & 6,94 & 11.100 .718 & 8,09 & 0,44 \\
\hline hijos de José Sabaler & Tarragona (Reus) & - & 1.109 .909 & 8,58 & $8.587,696$ & 12,92 & 0,54 \\
\hline Mangrané, Daniel & Barcelona & - & 1.305 .241 & 10,09 & 1.463 .661 & 89,18 & 0,64 \\
\hline Pallarés, Femando & Barcelona & 1932 & 1.084 .939 & 8,38 & 3.057 .679 & 35,48 & 0,53 \\
\hline Pedrol, Manuel & Тапаgona & - & 326.471 & 2,52 & 5.647 .091 & 5,78 & 0,16 \\
\hline Total Calaluña & - & - & 8.393 .980 & 64,86 & 46.500 .592 & 18,05 & 4,09 \\
\hline Cía. Exporladora Española & Madrid & 1933 & 34.572 & 0,27 & 5.822 .674 & 0,59 & 0,02 \\
\hline Salgado y Cía. & Madrid & 1920 & 21.751 & 0,17 & 2.857 .996 & 0,76 & 0,01 \\
\hline Total Madrid & - & - & 56.323 & 0,44 & 8.680 .670 & 0,65 & 0,03 \\
\hline Ballester y Cía. & Valencia & - & 2.225 & 0,02 & 1.764 .057 & 0,13 & 0,00 \\
\hline Total Valencia & - & - & 2.225 & 0,02 & 1.764 .057 & 0,13 & 0,00 \\
\hline TOTAL & - & - & 12.942 .344 & 100,00 & 205.321 .475 & 6,30 & 6,30 \\
\hline
\end{tabular}

Fuente: Resumen de las declaraciones de exportación presentadas a la F.E.A.O.E., Informe 2.539.

1: Provincia donde radica, 2: Año de constitución, 3: Volumen exportado, en Kgrs., 4: \% sobre el aceite exportado a Noruega, 5: Exportación al resto de los mercados, en Kgrs., 6: \% sobre el total exportado por la empresa a todos los mercados, 7: \% sobre el total de las exportaciones de todas las empresas. 
y por las características de sus socios, poco tienen que ver con las empresas qu se habían formado en el sector del cultivo del olivar o de la fabricación del acei $\mathrm{te}^{42}$. El elevado volumen de exportación alcanzado por alguna de estas empresa andaluzas, de fecha de constitución muy próxima al período al que se refieren lo. datos -Dulken, R.C.; Maestroni, S.A.; Pallares Hermanos, S.A.; Minerva, S.A. Moro, S.A.; Riva Hermanos, S.A.; La Utrerana, S.A.-, nos sugiere que una part significativa de los empresarios agrupados en ellas habrían de tener una important experiencia en el sector. Es decir, nuevas empresas, con nueva ubicación, consti tuidas por «viejos empresarios», procedentes, en muchos casos, de otras regione: con más experiencia en el sector.

Las diferencias entre las empresas catalanas y andaluzas se hacen más eviden tes si, en vez de referimos al volumen exportado a Noruega (cuadro 7, columna: 3 y 4), prestamos atención al significado de esas exportaciones en relación con l: actividad total de cada una de estas empresas (cuadro 7, columnas 5, 6 y 7). Er este caso, se constata un nivel de concentración mucho mayor en las catalanas qú en las andaluzas. Para estas últimas el mercado noruego supondría solamente e $3,03 \%$ o el $2,06 \%$, en función del indicador que tomemos de referencia. Para la: catalanas, el porcentaje se eleva al $18,05 \%$ y el $3,85 \%$, respectivamente. Po: empresas, en Andalucía sólo hay tres en las que el mercado noruego tenía un pesc significativo, Maestroni, S.A., (19,59\%), Pallares Hermanos, S.A. $(8,76 \%)$, y Lc Utrerana, S.A. (7,37\%). Las tres son empresas de reciente creación, en las que e empresariado de origen catalán tenía una importante presencia ${ }^{43}$. Por el contrario en un número importante de empresas catalanas el mercado noruego era un inte. resante destino.

\section{LA EXPORTACIÓN ESPAÑOLA A LOS MERCADOS MÁS IMPORTANTES}

A la luz de estos datos consideramos que es de interés establecer un contraste entre la situación del mercado noruego y otros mercados importantes para el aceite de oliva español. Los más de 70 mercados con los que estas empresas mante-

42. Una caracterización del empresariado agricola y fabricantes de aceite de oliva, en este caso referida sólc a la provincia de Jaén, podemos encontrarla en Hernández, S. (1997). pp. 338-356.

43. De estas tres empresas la primera en establecerse fue Pallares Hermanos. Sociedad Regular Colectiva De origen catalán, de Tortosa, se estableció en Cabra en 1917 con un capital social de 500.000 ptas. En 1928 st transformaría en sociedad anónima ampliando su capital hasta 7.000.000 de Ptas. La segunda de estas empresas La Utrerana, S.A., fue creada en 1923. Entre sus socios figuraba el barcelonés Daniel Mangrane Bocardo, de la empresa Daniel Mangrané, de Barcelona, que aportó la mayor parte del capital social de la empresa en el momentc de la constitución (1.250.000 Ptas., de un total de 1.300.000). En posteriores ampliaciones entrarian a formar parte de la sociedad nnevos empresarios catalanes. Este es el caso de los miembros de la conocida familia de exportadores de aceite de Tortosa: Francisco y Pedro Bau Blasi, o de Francisco Bau Nolla. La empresa Maestroni, S.A., se cré en 1926. Entre sus socios figuran de nuevo los hermanos Pallares. 
nían relaciones comerciales los hemos reducido a los más significados, 9 en total. A su vez, éstos los hemos agrupado en tres bloques: EE.UU., América Latina (Argentina, Cuba y Uruguay) y Europa (Francia, Inglaterra, Italia, Portugal y Noruega).

El escenario dibujado por los cuadros 8 y 9 es considerablemente distinto al que encontrábamos en el mercado noruego. En este caso el mayor volumen de exportación era canalizado a través de empresas radicadas en Andalucía. La proporción casi se invierte, un $70,58 \%$ de Andalucía, frente a un $25,19,85 \%$ de Cataluña ${ }^{44}$ (Cuadro 9, total de exportación, columna C), cuotas de mercado muy proporcionadas con la capacidad de producción de aceite en las dos regiones, ya que -entre 1930 y 1935 - el aceite andaluz suponía el $62 \%$ del total español ${ }^{45}$.

Los cuadros 8 y 9 nos permiten obtener algunas valoraciones más sobre el significado de las empresas exportadoras en ambas regiones. Del cuadro 8, se deduce que en las dos regiones la concentración de las ventas en determinadaos mercados se incrementa en relación inversa al tamaño de la empresa ${ }^{46}$. Sin embargo, la concentración era mayor en las empresas catalanas que en las radicada en Andalucía. En el primer caso, los índices son elevados incluso en las empresas con gran volumen de exportación ${ }^{47}$. Por el contrario, en el caso andaluz, salvo Longoria, S.C. (índice de concentración: 1,1509) y Olivarera Peninsular, S.A. (1,3112), el resto de las grandes empresas presentan una mayor diversificación en el destino de sus ventas.

44. Las exportaciones declaradas por estas empresas, para el período de 1930-julio de 1935. equivalen al $69.75 \%$ del total de las exportaciones españolas de aceite de oliva registrada por la Dirección General de Aduanas durante ese mismo periodo. Según cstas dos fuente, los países donde estas empresas canalizarían un menor volumen de la exportación española scría: Italia, donde controlarian el $77,93 \%$ y, sobre todo, Francia, con el 35,22 \%. Los datos de la exportación española por las diferentes aduanas los hemos obtenido en Zambrana. Fco. (1987), pp. $431-4.36$.

45. Zambrana, Fco. (1999).

46. En este caso el indicador de tamaño de la empresa sería el volumen de sus exportaciones.

47. Se define el "índice de concentración" por destinos de las exportaciones de accite de la empresa j como la suma, en valores absolutos, de todas las diferencias entre la participación individual y media de las exportaciones hacia cada pais. La medida que resulta pucde tomar valores entre 0 y 2 , de forma que, un índice elcvado un alto grado de especialización de la empresa en deteminados destinos. Si denotamos:

Eij = Exportaciones de la empresa $\mathrm{j}$ hacia el país $\mathrm{i}$

Ei. = Exportaciones de todas las empresas hacia el país $\mathrm{i}$

E. $\mathrm{j}=$ Exportaciones de la empresa $\mathrm{j}$

$\mathrm{E}=$ Exportaciones totales de las empresas recogidas

Rij $=$ Eij/E.j = Participación del país $\mathrm{i}$ en las exportaciones de la empresa $\mathrm{j}$

$\mathrm{Rji}=\mathrm{Eij} /$ Ei. $=$ Participación de la empresa $\mathrm{j}$ en las exportaciones hacia el país $\mathrm{i}$

$\mathrm{Ri} .=\mathrm{Ei} . / \mathrm{E}=$ Participación del país $\mathrm{i}$ en las exportaciones totales

$R . j=E . j / E=E . j / E=$ Participación de la empresa $\mathrm{j}$ en las exportaciones totales

El índice de concentración por destinos resulta:

$L j=\sum_{t} / R_{y}-R i . /=$ Indice de concentración en los destinos de la empresa j 
CUADRO 8

EXPORTACIÓN DE ACEITE DE OLIVA A LOS PRINCIPALES MERCADOS, 1930-JULIO 1935 (EN TNS.)

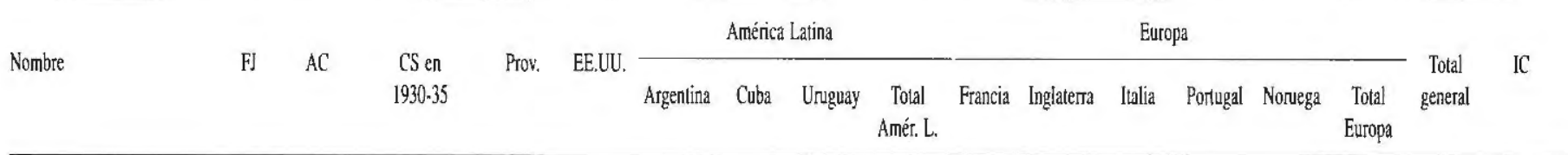

\begin{tabular}{|c|c|c|c|c|c|c|c|c|c|c|c|c|c|c|c|c|c|}
\hline \multicolumn{18}{|l|}{ Radicadas en Andalucía } \\
\hline Carbonell (Córdoba) & S.C. & 1896 & 10.000 .000 & $\mathrm{CO}$ & 14.935 & 1.873 & 5.256 & 2.105 & 9.234 & 3 & 904 & 2.106 & 3.300 & 1.244 & 7.558 & 31.726 & 0,62 \\
\hline Minerva, S.A. & S.A. & 1919 & 1.000 .000 & MA & 11.179 & 1.320 & 523 & 82 & 1.925 & 63 & 642 & 13.548 & 1.829 & 337 & 16.419 & 29.523 & 0,88 \\
\hline Ybarra (Hijos de) & S.C. & 1885 & 19.992 .000 & SE & 6.594 & 14.569 & 160 & 5.562 & 20.291 & 0 & 80 & 0 & 750 & 61 & 891 & 27.776 & 0,90 \\
\hline Longona (Miguel G.) & S.C. & 1909 & 2.796 .720 & SE & 96 & 12.248 & 5.759 & 6.142 & 24.149 & 0 & 0 & 0 & 0 & 0 & 0 & 24.234 & 1,15 \\
\hline Moro, S.A. & S.A. & 1926 & 4.004 .000 & $\mathrm{MA}$ & 1.833 & 2.802 & 962 & 1.206 & 4,970 & 41 & 46 & 11.281 & 1.096 & 322 & 12.786 & 19.590 & 0,82 \\
\hline Dulken (E. Van) & R.C. & 1932 & 100.000 & MA & 4.750 & 21 & 800 & 0 & 821 & 80 & 3.893 & 1.878 & 256 & 392 & 6.499 & 12,070 & 0,85 \\
\hline Olivarera Peninsular, S.A. & S.A. & 1931 & 1.010 .000 & MA & 7.516 & 0 & 64 & 0 & 64 & 0 & 229 & 596 & 0 & 0 & 825 & 8.405 & 1,31 \\
\hline Pallarés Hermanos, S.A. & R.C. & 1917 & 7.000 .000 & $\mathrm{CO}$ & 2.732 & 136 & 12 & 36 & 185 & 71 & 95 & 2.327 & 1.968 & 708 & 5.169 & 8.086 & 0,91 \\
\hline Torres y Ribellas & R.C. & 1914 & 512.772 & SE & 322 & 2.084 & 1.399 & 3.882 & 7.364 & 0 & 91 & 0 & 196 & 0 & 287 & 7.974 & 1,01 \\
\hline Maestroni, S.A. & S.A. & 1926 & 1.010 .000 & MA & 1.699 & 56 & 14 & 0 & 70 & 403 & 482 & 2.242 & 559 & 1.329 & 5.015 & 6.784 & 0,82 \\
\hline Riva Hermanos & S.A. & 1926 & 1.005 .000 & MA & 2.215 & 8 & 2.583 & 0 & 2.591 & 19 & 337 & 587 & 585 & 76 & 1.604 & 6.411 & 0,82 \\
\hline Luca de Tena (Hijos de) & R.C. & 1891 & 4.040 .000 & SE & 697 & 1.591 & 2.280 & 16 & 3.887 & 57 & 916 & 54 & 590 & 5 & 1.621 & 6.206 & 0,84 \\
\hline Bruguer y Trujillo & - & 1923 & 480.000 & SE & 676 & 8 & 4.990 & 0 & 4.998 & 0 & 6 & 0 & 4 & 0 & 10 & 5.683 & 1,46 \\
\hline Carbonell (Sevilla) & - & - & - & SE & 3 & 0 & 5.033 & 0 & 5.033 & 0 & 0 & 0 & 0 & 0 & 0 & 5.036 & 1,71 \\
\hline Francés y Cía. & OT & 1882 & 2.000 .000 & $\mathrm{CO}$ & 0 & 1.406 & 35 & 0 & 1.441 & 0 & 0 & 0 & 548 & 0 & 548 & 1.988 & 1,46 \\
\hline Olivarera del Mediter. & S.A. & 1928 & 2.000 .000 & MA & 112 & 0 & 0 & 0 & 0 & 0 & 0 & 1.703 & 0 & 0 & 1.703 & 1.815 & 1,53 \\
\hline Espuny, Agustín & - & - & - & $\mathrm{JA}$ & 0 & 0 & 0 & 0 & 0 & 875 & 0 & 708 & 0 & 0 & 1.583 & 1.583 & 1,60 \\
\hline Aceitera del Mediter. & S.A. & 1917 & 1.005 .000 & MA & 104 & 0 & 832 & 0 & 832 & 0 & 4 & 0 & 0 & 0 & 4 & 940 & 1,48 \\
\hline Funes e Hijos & - & - & - & $\mathrm{JA}$ & 1 & 0 & 8 & 0 & 8 & 0 & 0 & 833 & 0 & 0 & 833 & 842 & 1,63 \\
\hline Monturque, S.A. & S.A. & 1923 & 700.000 & JA & 0 & 0 & 12 & 0 & 12 & 0 & 0 & 256 & 0 & 0 & 256 & 267 & 1,57 \\
\hline Jiménez y Cía., Pablo & S.C. & 1903 & 900.000 & JA & 0 & 15 & 33 & 0 & 48 & 11 & 0 & 123 & 25 & 0 & 159 & 207 & 1,07 \\
\hline Utrerana, S.A., La & S.A. & 1923 & 2.100 .000 & SE & 18 & 0 & 0 & 0 & 0 & 89 & 1 & 69 & 13 & 15 & 187 & 205 & 1,23 \\
\hline Larios, S.A. & S.A. & 1933 & 10.000 .000 & MA & 0 & 12 & 0 & 0 & 12 & 0 & 0 & 0 & 19 & 0 & 19 & 31 & 1,50 \\
\hline Rodríguez Hemnanos & R.C. & 1887 & 10.000 & $\mathrm{CO}$ & 0 & 0 & 0 & 0 & 0 & 0 & 0 & 0 & 10 & 0 & 10 & 10 & 1,91 \\
\hline Oleum, S.A. & S.A. & 1920 & 2.100 .00 & JA & 0 & 0 & 0 & 0 & 0 & 0 & 0 & 0 & 0 & 0 & 0 & 0 & 0 \\
\hline
\end{tabular}


CUADRO 8 (Continuación)

\begin{tabular}{|c|c|c|c|c|c|c|c|c|c|c|c|c|c|c|c|c|c|}
\hline \multirow{2}{*}{ Nombre } & \multirow{2}{*}{$\mathrm{FJ}$} & \multirow{2}{*}{$\mathrm{AC}$} & \multirow{2}{*}{$\begin{array}{c}C S \text { en } \\
1930-35\end{array}$} & \multirow{2}{*}{ Prov. } & \multirow{2}{*}{ EE.UU. } & \multicolumn{4}{|c|}{ América Latina } & \multicolumn{6}{|c|}{ Europa } & \multirow{2}{*}{$\begin{array}{c}\text { Total } \\
\text { general }\end{array}$} & \multirow{2}{*}{ IC } \\
\hline & & & & & & Argentina & Cuba & Uruguay & $\begin{array}{c}\text { Total } \\
\text { Amér. L. }\end{array}$ & Francia & Inglaterra & Italia & Portugal & Noruega & $\begin{array}{c}\text { Tolal } \\
\text { Europa }\end{array}$ & & \\
\hline \multicolumn{18}{|l|}{ Radicadas en Cataluña } \\
\hline Bau, S.A. & S.A. & 1890 & $3.000 .000(*)$ & $\mathrm{TA}$ & 414 & 11.597 & 1.226 & 1.246 & 14.069 & 0 & 57 & 0 & 0 & 0 & 57 & 14,540 & 1,22 \\
\hline Gasull (Est. Félix) & S.A. & 1922 & $2.000 .000\left({ }^{*}\right)$ & $\mathrm{TA}$ & 2.847 & 3 & 1.779 & 9 & 1.791 & 1.439 & 665 & 3.384 & 77 & 898 & 6.463 & 11.101 & 0,63 \\
\hline Sensat e Hijos (G.) & R.C. & 1878 & $9.900 .000\left(^{*}\right)$ & $\mathrm{BA}$ & 642 & 953 & 7.633 & 383 & 8.968 & 0 & 4 & 0 & 0 & 0 & 4 & 9.614 & 1,30 \\
\hline Ballester Romero, Juan & - & - & - & $\mathrm{TA}$ & 282 & 3.814 & 0 & 23 & 3.836 & 104 & 5 & 1.873 & 48 & 2.799 & 4.830 & 8.948 & 1,05 \\
\hline Hijos de José Sabater & - & - & - & $\mathrm{TA}$ & 624 & 4.036 & 378 & 84 & 4.498 & 530 & 974 & 851 & 0 & 1.110 & 3.465 & 8.588 & 0,90 \\
\hline Fontana, Enrique & - & - & - & $\mathrm{TA}$ & 1.614 & 379 & 3 & 0 & 382 & 866 & 2.756 & 918 & 0 & 113 & 4.652 & 6.648 & 0,95 \\
\hline Pedrol, Manuel & - & - & - & $\mathrm{TA}$ & 100 & 268 & 262 & 9 & 539 & 1.355 & 1.628 & 1.699 & 0 & 326 & 5.009 & 5.647 & 1,19 \\
\hline Pallarés, Fernando & S.A. & 1932 & $1.355 .000\left(^{*}\right)$ & $\mathrm{BA}$ & 101 & 361 & 161 & 59 & 50 & 420 & 333 & 472 & 66 & 1.085 & 2.376 & 3.058 & 0,96 \\
\hline Panisello, Juan & - & - & - & $\mathrm{TA}$ & 539 & 0 & 0 & 0 & 0 & 390 & 55 & 1.401 & 0 & 0 & 1.845 & 2.384 & 1,11 \\
\hline Mangrané, Daniel & - & - & $3.050 .000(*)$ & $\mathrm{BA}$ & 2 & 29 & 2 & 0 & 30 & 29 & 14 & 84 & 0 & 1.305 & 1.431 & 1.464 & 1,70 \\
\hline Basseda, Antonia & - & 1850 & - & $\mathrm{BA}$ & 79 & 89 & 0 & 27 & 116 & 0 & 4 & 90 & 0 & 758 & 853 & 1.048 & 1,36 \\
\hline Armengol, S.A. & - & - & - & $\mathrm{BA}$ & 0 & 4 & 368 & 0 & 372 & 0 & 0 & 0 & 0 & 0 & 0 & 372 & 1,69 \\
\hline Fabril y Comer. Balcells & - & - & - & $\mathrm{BA}$ & 0 & 0 & 302 & 0 & 302 & 23 & 0 & 0 & 0 & 0 & 23 & 325 & 1,66 \\
\hline Aceites Pocar, S.A. & S.A. & 1857 & $2.010 .00(*)$ & $\mathrm{BA}$ & 0 & 69 & 5 & 5 & 78 & 16 & 1 & 171 & 0 & 0 & 188 & 267 & 1,11 \\
\hline Autran Barberán, Fdo. & - & - & - & $\mathrm{BA}$ & 0 & 12 & 0 & 0 & 12 & 0 & 0 & 0 & 0 & 0 & 0 & 12 & 1,59 \\
\hline Salat, S.A. & S.A. & 1925 & $2.000 .000(*)$ & $\mathrm{BA}$ & 0 & 1 & 0 & 0 & 1 & 0 & 0 & 0 & 0 & 0 & 0 & 1 & 1,45 \\
\hline La Aceitera Exportad. & S.A. & 1913 & $1.500 .000\left(^{*}\right)$ & $\mathrm{BA}$ & 0 & 0 & 0 & 0 & 0 & 0 & 0 & 0 & 0 & 0 & 0 & 0 & 0 \\
\hline Total Cataluña & - & - & - & - & 7.243 & 21.613 & 12,119 & 1.843 & 35.575 & 5.172 & 6.496 & 10.944 & 192 & 8.394 & 31.197 & 74.015 & $\begin{array}{c}0,52 \\
(2) 1,24\end{array}$ \\
\hline
\end{tabular}

(*) En 1936. 


\section{CUADRO 8 (Continuación)}

\begin{tabular}{|c|c|c|c|c|c|c|c|c|c|c|c|c|c|c|c|c|c|}
\hline \multirow{2}{*}{ Vombre } & \multirow{2}{*}{ FJ } & \multirow{2}{*}{$\mathrm{AC}$} & \multirow{2}{*}{$\begin{array}{l}\text { CS en } \\
1930-35\end{array}$} & \multirow{2}{*}{ Prox. } & \multirow{2}{*}{ EE.UU. } & \multicolumn{4}{|c|}{ América Latina } & \multicolumn{6}{|c|}{ Europa } & \multirow{2}{*}{$\begin{array}{c}\text { Total } \\
\text { general }\end{array}$} & \multirow{2}{*}{ IC } \\
\hline & & & & & & Argentina & Cuba & Unug̣uay & $\begin{array}{l}\text { Total } \\
\text { Amér. L. }\end{array}$ & Francia & Inglalerra & Italia & Potugal & Noruega & $\begin{array}{l}\text { Total } \\
\text { Europa }\end{array}$ & & \\
\hline $\begin{array}{l}\text { Oleivinicola del C. } \\
\text { de España, S.A. }\end{array}$ & - & - & - & CR & 0 & 0 & 0 & 0 & 0 & 268 & 0 & 7 & 1.725 & 0 & 2.000 & 2.000 & 1,85 \\
\hline Colomer & S.A. & 1933 & $500.000\left({ }^{*}\right)$ & M & 0 & 0 & 0 & 0 & 0 & 0 & 0 & 0 & 0 & 0 & 0 & 0 & 0 \\
\hline Salgado y Cía. & S.A. & 1920 & $3.000 .000\left(^{*}\right)$ & M & 1.858 & 161 & 9 & 9 & 161 & 2 & 9 & 574 & 241 & 22 & 738 & 2.757 & 9.85 \\
\hline Cía. Export. Española & - & 1933 & - & $\mathrm{M}$ & 5.237 & 0 & 0 & 0 & 0 & 0 & 526 & 25 & 0 & 35 & 586 & 5.823 & 1,40 \\
\hline Total Madrid & - & - & - & - & 7.096 & 161 & 0 & 0 & 161 & 2 & 626 & 599 & 241 & 56 & 1.424 & 8.681 & 1,18 \\
\hline Ballester y Cía. & - & - & - & $V A$ & 323 & 51 & 0 & 0 & 51 & 29 & 88 & 1.270 & 0 & 2 & 1.390 & 1.764 & 1,09 \\
\hline Casanova, S.A. & S.A. & 1921 & $3.000 .000\left(^{*}\right)$ & VA & 0 & 0 & 0 & 0 & 0 & 0 & 0 & 0 & 0 & 0 & 0 & 0 & 0 \\
\hline Total Valencia & - & - & - & - & 323 & 51 & $\theta$ & 0 & 51 & 29 & 88 & 1.270 & 0 & 2 & 1,390 & 1.764 & 1,09 \\
\hline TOTAL GENERAL & - & - & - & - & 70.136 & 59.975 & 42.881 & 20.873 & 123.719 & 7.184 & 14.836 & 51.132 & 13.904 & 12.942 & 99.998 & 293.863 & - \\
\hline
\end{tabular}

(*) En 1936 .

Fuente: Resumen de las declaraciones de exportación presentadas a la F.E.A.0.E., Informe 2.539.

(1) Media aritmética andaluza. (2) Media aritmética catalana, FJ: Forma juridica al constituirse, AC: Año de constitución. CS: Capital social, IC: Índice de concentración. 
Las diferencias aumentan si prestamos atención al cuadro 9. En él hemos agrupado, como se indicó anteriormente, los nueve principales mercados en tres grandes áreas: EE.UU., América Latina y Europa. Como podemos observar por la importancia de las exportaciones de cada región a los diferentes ámbitos, columna $\mathrm{C}$, las empresas catalanas, en su conjunto, tenían una presencia similar en los mercados de Europa y América del Sur; y, en tercer lugar, a gran distancia de los anteriores, en los EE.UU. Una imagen distinta presentan las empresas andaluzas, tomadas en su conjunto.

En un análisis detallado, prestando atención a cada una de las empresas de las dos regiones, columna B, se confirma esta impresión general. Pues, aunque encontramos algunas grandes empresas catalanas que concentraban el grueso de su negocio con América Latina, mercado en el que tendrían un gran arraigo y prestigio $^{48}$, caso de Bau S.A., o Sensat e hijos, la mayoría de ellas dirigen el mayor porcentaje de sus exportaciones al mercado europeo. En el caso andaluz, aunque, asimismo, había un nutrido grupo, de entre las grandes empresas, que tenía en Europa un importante destino de sus ventas, son los EE.UU. y Latino América sus grandes mercados.

Conocer las causas de esta distribución de las exportaciones en las dos grandes regiones productoras requeriría estudios específicos sobre cada uno de estos mercados $^{49}$. A nuestro entender, aunque sólo sea una hipótesis sin contrastar, el predominio de los exportadores catalanes en Europa estaría relacionado, por un lado, con una demanda más exigente, que busca aceites de mayor calidad, condición que cumplían un porcentaje importante de los aceites catalanes, sobre todo los de las comarcas leridanas de Borjas Blancas. Este sería el caso de gran parte de los aceites destinados a Francia, Italia, para la realización de «coupage», y a la industria conservera noruega ${ }^{50}$. Por otro, con la mayor tradición comercial y conocimiento del mercado europeo que caracterizaba al empresariado catalán.

Por el contrario, al mercado americano, tanto del norte como del sur, con una demanda menos exigente, se enviaban grandes partidas de aceite andaluz, de calidad inferior, mezcla de refinado, un $70 \%$, y virgen, un $30 \%$, pero a un precio también más bajo ${ }^{51}$. El aceite andaluz se enviaba, así mismo, a los países intermediarios: Francia y, sobre todo, Italia, desde donde, tras ser refinado y mezclado, se comercializaba, bajo marcas de estos países, en los más importantes mercados.

48. Algunas notas sobre la presencia de estos empresarios en América podemos encontrarlas en Ramón, R. (1996). p. 50 .

49. Un estudio sobre las características de los diferentes mercados podemos encontrarlo en: Ramón, R. (1999).

50. Sobre la exportación de aceite catalán a países europeos para la realización de «coupages», ver: Gaya, M. (1930), p. 11, Mathiesen, E. (1933).

51. Mathiesen, E. (1933). 
CUADRO 9

PRINCIPALES ÁMBITOS DE EXPORTACIÓN DE LAS EMPRESAS ESPAÑOLAS DEL SECTOR DEL ACEITE DE OLIVA, 1930-JULIO 1935 (EN TONELADAS)

\begin{tabular}{|c|c|c|c|c|c|c|c|c|c|c|c|}
\hline \multirow{2}{*}{ Nombre } & \multicolumn{3}{|c|}{ EE.UU. } & \multicolumn{3}{|c|}{ América Lalina } & \multicolumn{3}{|c|}{ Europa } & \multicolumn{2}{|c|}{ Total general } \\
\hline & A & B & $\mathrm{C}$ & A & $\mathrm{B}$ & $\mathrm{C}$ & A & B & $\mathrm{C}$ & A & $\mathrm{C}$ \\
\hline \multicolumn{12}{|l|}{ Radicadas en Andalucía } \\
\hline Carbonell (Córdoba) & 14.935 & 47,07 & 21,29 & 9.234 & 29,10 & 7,46 & 7.558 & 23,82 & 7,56 & 31.726 & 10,80 \\
\hline Minerva, S.A. & 11.179 & 37,87 & 15,94 & 1.925 & 6,52 & 1,56 & 16.419 & 55,61 & 16,42 & 29.523 & 10,05 \\
\hline Ibarra (Hijos de) & 6.594 & 23,74 & 9,40 & 20.291 & 73,05 & 16,40 & 891 & 3,21 & 0,89 & 27.776 & 9,45 \\
\hline Longoria (Miguel G.) & 86 & 0,35 & 0,12 & 24.149 & 99,65 & 19,52 & 0 & 0,00 & 0,00 & 24.234 & 8,25 \\
\hline Moro, S.A. & 1.833 & - & 2,61 & 4.970 & 25,37 & 4,02 & 12.786 & 65,27 & 12,79 & 19.590 & 6,67 \\
\hline Dulken (E. Van) & 4.750 & 39,35 & 6,77 & 821 & 6,80 & 0,66 & 6.499 & 53,85 & 6,50 & 12.070 & 4,11 \\
\hline Olivarera Peninsular, S.A. & 7.516 & 89,43 & 10,72 & 64 & 0,76 & 0,05 & 825 & 9,82 & 0,83 & 8.405 & 2,86 \\
\hline Pallarés Hermanos & 2.732 & 33,78 & 3,89 & 185 & 2,28 & 0,15 & 5.169 & 63,93 & 5,17 & 8.086 & 2,75 \\
\hline Torres y Ribellas & 322 & 4,94 & 0,46 & 7.364 & 92,36 & 5,95 & 287 & 3,60 & 0,29 & 7.974 & 2,71 \\
\hline Maestroni, S.A. & 1.699 & 25,05 & 2,42 & 70 & 1,03 & 0,06 & 5.015 & 73,93 & 5,02 & 6.784 & 2,31 \\
\hline Riva Hermanos & 2.215 & 34,56 & 3,16 & 2.591 & 40,42 & 2,09 & 1.604 & 25,02 & 1,60 & 6.411 & 2,18 \\
\hline Luca de Tena (Hijos de) & 697 & 11,23 & 0,99 & 3.887 & 62,64 & 3,14 & 1.621 & 26,13 & 1,62 & 6.206 & 2,11 \\
\hline Bruguier y Trujillo & 676 & 11,89 & 0,96 & 4.998 & 87,93 & 4,04 & 10 & 0,18 & 0,01 & 5.683 & 1,93 \\
\hline Carbonell (Sevilla) & 3 & 0,06 & 0,00 & 5.033 & 99,94 & 4,07 & 0 & 0,00 & 0,00 & 5.036 & 1,71 \\
\hline Francés y Cía. & 0 & 0,00 & 0,00 & 1.441 & 72,46 & 1,16 & 548 & 27,54 & 0,55 & 1.988 & 0,68 \\
\hline Olivarera del Mediterráneo & 112 & 6,18 & 0,16 & 0 & 0,02 & 0,00 & 1.703 & 93,80 & 1,70 & 1.815 & 0,62 \\
\hline Espuny, Agustín & 0 & 0,00 & 0,00 & 0 & 0,00 & 0,00 & 1.583 & 100,0 & 1,58 & 1.583 & 0,54 \\
\hline Aceitera del Mediteráneo & 104 & 11,09 & 0,15 & 832 & 88,50 & 0,67 & 4 & 0,41 & 0,00 & 940 & 0,32 \\
\hline Funes e Hijos & 1 & 0,07 & 0,00 & 8 & 1,01 & 0,01 & 833 & 98,93 & 0,83 & 842 & 0,29 \\
\hline Monturque, S.A. & 0 & 0,00 & 0,00 & 12 & 4,30 & 0,01 & 256 & 95,70 & 0,26 & 267 & 0,09 \\
\hline Jiménez y Cía., Pablo & 0 & 0,00 & 0,00 & 48 & 23,15 & 0,04 & 159 & 76,85 & 0,16 & 207 & 0,07 \\
\hline Utrerana, S.A., La & 18 & 8,87 & 0,03 & 0 & 0,00 & 0,00 & 187 & 91,13 & 0,19 & 205 & 0,07 \\
\hline Larios, S.A. & 0 & 0,00 & 0,00 & 12 & 38,83 & 0,01 & 19 & 61,17 & 0,02 & 31 & 0,01 \\
\hline Gross Hermanos & 2 & 22,35 & 0,00 & 8 & 77,65 & 0,01 & 0 & 0,00 & 0,00 & 11 & 0,00 \\
\hline Rodríguez Hemlanos & 0 & 0,00 & 0,00 & 0 & 0,00 & 0,00 & 10 & 100,00 & 0,01 & 10 & 0,00 \\
\hline Oleum, S.A. & 0 & - & 0,00 & 0 & - & 0,00 & 0 & - & 0,00 & 0 & 0,00 \\
\hline
\end{tabular}


CUADRO 9 (Continuación)

\begin{tabular}{|c|c|c|c|c|c|c|c|c|c|c|c|}
\hline \multirow{2}{*}{ Nombre } & \multicolumn{3}{|c|}{ EE.UU. } & \multicolumn{3}{|c|}{ América Latina } & \multicolumn{3}{|c|}{ Europa } & \multicolumn{2}{|c|}{ Tolal general } \\
\hline & A & $B$ & $\mathrm{C}$ & A & B & $\mathrm{C}$ & A & B & $\mathrm{C}$ & A & $\mathrm{C}$ \\
\hline \multicolumn{12}{|l|}{ Radicadas en Cataluña } \\
\hline Bau, S.A. & 414 & 2,85 & 0,59 & 14.069 & 96,76 & 11,37 & 57 & 0,39 & 0,06 & 14.540 & 4,95 \\
\hline Gasull (Est. Félix) & 2.847 & 25,65 & 0,06 & 1.791 & 16,13 & 1,45 & 6.463 & 58,22 & 6,46 & 11.101 & 3,78 \\
\hline Sensat e Hijos (G.) & 642 & 6,67 & 0,91 & 8.968 & 93,28 & 7,25 & 4 & 0,04 & 0,00 & 9.614 & 3,27 \\
\hline Ballester Romero, Juan & 282 & 3,15 & 0,40 & 3.836 & 42,87 & 3,10 & 4.830 & 53,97 & 4,83 & 8.948 & 3,04 \\
\hline Hijos de José Sabaler & 624 & 7,27 & 0,89 & 4.498 & 52,38 & 3,64 & 3.465 & 40,35 & 3,47 & 8.588 & 2,92 \\
\hline Fontana, Enrique & 1.614 & 24,27 & 2,30 & 382 & 5,75 & 0,31 & 4.652 & 69,98 & 4,65 & 6.648 & 2,26 \\
\hline Pedrol, Manuel & 100 & 1,77 & 0,14 & 539 & 9,54 & 0,44 & 5.009 & 88,69 & 5,01 & 5.647 & 1,92 \\
\hline Pallarés, Femando & 101 & 3,31 & 0,14 & 580 & 18,98 & 0,47 & 2.376 & 77,71 & 2,38 & 3.058 & 1,04 \\
\hline Panisello, Juan & 539 & 22,60 & 0,77 & 0 & 0,00 & 0,00 & 1.845 & 77,40 & 1,85 & 2.384 & 0,81 \\
\hline Mangrané, Daniel & 2 & 0,13 & 0,00 & 30 & 2,08 & 0,02 & 1.431 & 97,80 & 1,43 & 1.464 & 0,50 \\
\hline Basseda, Antonia & 79 & 7,56 & 0,11 & 116 & 11,06 & 0,09 & 853 & 81,37 & 0,85 & 1.048 & 0,36 \\
\hline Armengol, S.A. & 0 & 0,00 & 0,00 & 372 & 100,00 & 0,30 & 0 & 0,00 & 0,00 & 372 & 0,13 \\
\hline Fabril y Comercial Balcells & 0 & 0,00 & 0,00 & 302 & 92,79 & 0,24 & 23 & 7,21 & 0,02 & 325 & 0,11 \\
\hline Aceites Pocar, S.A. & 0 & 0,00 & 0,00 & 78 & 29,37 & 0,06 & 188 & 70,63 & 0,19 & 267 & 0,09 \\
\hline Aulran Barberán, Femaando & 0 & 0,00 & 0,00 & 12 & 100,00 & 0,01 & 0 & 0,00 & 0,00 & 12 & 0,00 \\
\hline La Aceitera Exportadora & 0 & - & 0,00 & 0 & - & 0,00 & 0 & - & 0,00 & 0 & 0,00 \\
\hline Salat, S.A. & 0 & 0,00 & 0,00 & 1 & 100,00 & 0,00 & 0 & 0,00 & 0,00 & 1 & 0,00 \\
\hline Total Cataluña & 7.243 & - & 10,33 & 35.575 & - & 28,75 & 31.197 & - & 31,20 & 74,015 & 25,19 \\
\hline
\end{tabular}




\section{CUADRO 9 (Continuación)}

\begin{tabular}{|c|c|c|c|c|c|c|c|c|c|c|c|}
\hline \multirow{2}{*}{ Nombre } & \multicolumn{3}{|c|}{ EE.UU. } & \multicolumn{3}{|c|}{ América Latina } & \multicolumn{3}{|c|}{ Europa } & \multicolumn{2}{|c|}{ Total general } \\
\hline & A & B & C & A & B & C & $\mathrm{A}$ & B & $\mathrm{C}$ & A & $\mathrm{C}$ \\
\hline Oleivinícola C. España, S.A. & 0 & - & 0,00 & 0 & - & 0,00 & 2,000 & - & 2,00 & 2.000 & 0,68 \\
\hline Colomer & 0 & - & 0,00 & 0 & - & 0,00 & 0 & - & 0,00 & 0 & 0,00 \\
\hline Salgado y Cía. & 1.858 & 65,02 & 2,65 & 161 & 5.63 & 0,13 & 839 & 29,34 & 0,84 & 2.858 & 0,97 \\
\hline Cía. Exporadora Española & 5.237 & 89.94 & 7.47 & 0 & 0.00 & 0,00 & 586 & 10,06 & 0,59 & 5.823 & 1.98 \\
\hline Total Madrid & 7.096 & - & 10,12 & 161 & - & 0,13 & 1.424 & - & 1,42 & 8.681 & 2,95 \\
\hline Ballester y Cía. & 323 & 18,33 & 0.46 & 51 & 2.91 & 0,04 & 1.390 & 78.77 & 1,39 & 1.764 & 0,60 \\
\hline Casanova, S.A. & 0 & - & 0,00 & 0 & - & 0,00 & 0 & - & 0.00 & 0 & 0,00 \\
\hline Total Valencia & 323 & - & 0.46 & 51 & - & 0,04 & 1.390 & - & 1,39 & 1.784 & 0,60 \\
\hline TOTAL GENERAL & 70.136 & - & 100,00 & 123.729 & - & 100,00 & 99.998 & - & 100,00 & 293.863 & 100,00 \\
\hline
\end{tabular}

Fuente: Cuadro 8.

A: Exportación en toneladas, B: \% sobre la exportación total de la empresa, C: \% sobre el total de las exporlaciones. 


\section{LAS EMPRESAS EXPORTADORAS DE ACEITE DE OLIVA EN ANDALUCÍA}

La cuestión en la que vamos a centrarnos a continuación gira en torno a cuáles fueron las empresas y quiénes los empresarios que, desde Andalucía, consiguieron esta progresiva evolución de la exportación del aceite de oliva. Sólo vamos a trazar unas suaves pinceladas sobre el perfil y las formas de organizar el negocio de unas empresas y empresarios, en su inmensa mayoría desconocidos ${ }^{52}$, que requieren un estudio más detallado.

La fuente fundamental es el Registro Mercantil de las ocho provincias andaluzas. Su información es clave pues, prácticamente, la totalidad de las empresas que venimos estudiando se organizaron bajo la forma de sociedad mercantil, en alguna de sus modalidades. Otras fuentes, como los Anuarios Financieros, podrían facilitarnos información al respecto, pero en este caso, no sólo perderíamos información sobre las empresas que adoptan formas diferentes a la de Sociedad Anónima, sino que, además, no tendríamos información de sus socios y de su evolución.

El primer hecho que llama la atención es el importante incremento en la constitución de empresas a partir de 1914. La Gran Guerra Europea debió influir, por causas de todos conocidas, en este auge. Asimismo, los años de tranquilidad y bonanza económica que experimentó el sector aceitero durante la dictadura de Primo de Rivera sería un factor a tener en cuenta. Sin embargo, la causa fundamental habría que buscarla en los avances experimentados en el refino ${ }^{53}$. De las 26 empresas exportadoras radicadas en Andalucía, 15, es decir el 57,69\%, se constituyeron con posterioridad a 1914.

En segundo lugar, destaca su ubicación. No cabe duda que el fuerte incremento de la producción y calidad del aceite en Andalucía debió estar presente en este empresariado a la hora de tomar una decisión al respecto. Ahora bien, dentro de este marco general, sería la disponibilidad de vías de transporte, la posibilidad de llegar fácilmente a los mercados, lo que determinará, en última instancia, el lugar donde se establecen unas empresas, exportadoras y refinadoras, cuya actividad no está determinada por la proximidad de la materia prima. Ello explicaría que la provincia de Jaén, la mayor productora de aceite de oliva, sea la que menor número de empresas registre. Por el contrario, fueron los puertos de mayor tradición exportadora, Málaga y Sevilla, o núcleos olivareros importantes, pero bien comu-

52. De la mayor parte de estas empresas y empresarios andaluces carecemos de información. Los únicos estudios detallados de los que disponemos son los de Rafael Castejón (1977), sobre la casa Carbonell, los de Francisco Zambrana sobre esta misma casa, aunque, en esta ocasión centrados sobre los aspectos de propietarios de olivar (1987), pp. 163-197 y los de María Sierra para los Ybarra. Algunas referencias sobre la familia Pallares podemos encontrarlas en Romero, T. (1996), pp. 457-460.

53. Sobre los origenes del proceso moderno del refino de los aceites de oliva, ver: Savastano, G. y Sericano, E. (1935), pp. 3-16. 
nicados por el llamado "ferrocarril del aceite $»^{54}$, los que acogieron a estas empresas. En estos núcleos se establecieron las refinerías y los grandes depósitos a los que llegaban, vía ferrocarril o en otros medios muchos más precarios cuando aquel no existía, los aceites del interior andaluz ${ }^{55}$.

En tercer lugar, las formas de organización y financiación. Al contrario que en el cultivo del olivar y fabricación del aceite, donde predominaba, por las débiles barreras de entrada, la empresa de carácter individual y de escasos recursos financieros, en el refino y exportación la asociación de capitales se hacía imprescindi$b^{5} e^{56}$. El refino, neutralización y desodorización, la elaboración, mediante mezclas, de un producto homogéneo, adaptado al gusto de los consumidores, el conocimiento de los mercados y un abastecimiento seguro de los mismos, requería disponer de un volumen importante de capital. Mientras el capital medio de las sociedades dedicadas a la fabricación de aceite se situaba en torno a las $179.613 \mathrm{Ptas}^{57}$, la media de las sociedades dedicadas a la exportación y, en muchos casos, también el refino, era de 2.957.522 Ptas.

En cuarto lugar, el perfil del empresario. Al contrario de lo que ocurría en el sector del cultivo y la fabricación, en el refino y comercialización del aceite de oliva andaluz no sobresalían los propietarios de olivares, la burguesía agrícola ${ }^{58}$. En el accionariado de estas empresas eran las profesiones relacionadas con el comercio y la industria las que predominaban (cuadro 10).

Es cierto que un porcentaje importante de los socios figuraban dentro de la categoría de "propietarios»; sin embargo, esta presencia debería matizarse al menos en un doble sentido. Por un lado, porque en las sociedades constituidas por socios «propietarios» y por socios «industriales» o «del comercio», el mayor esfuerzo financiero fue soportado por estos últimos. Asimismo, en muchos casos, eran los comerciantes e industriales, con frecuencia procedentes de regiones donde la burguesía industrial y comercial tiene una mayor implantación, los que toman la iniciativa; los "propietarios» se limitarían a apoyarla. Este es el caso de las citadas sociedades de: Pallares Hermanos, en sus diferentes formas jurídicas, La Utrerana, S.A., Maestroni, S.A., Dulken, S.A., Moro, S.A. o Espuny, entre otras. Por otro, habría que tener en cuenta, a la hora de conocer la verdadera procedencia sociológica de estos empresarios, la fuerte presión que la estructura social andaluza ejercía sobre ellos. Es decir, el hecho de que en la Andalucía del primer ter-

54. Se trata del ferrocarril Linares-Puente Genil, que daba salida a los aceites de las comarcas jiennenses y cordobasesas donde el olivar tenía mayor implantación. Enlazando con el ferrocarril de Córdoba a Málaga esta prodncción llegaba a su principal centro de exportación, el puerto de Málaga. Tedde, P. (1981), pp. 198-209. Sobre la importancia de la disponibilidad de vîas de comunicación en la localización de las empresas andaluzas de este período, ver: Castejón; R. (1977), pp. 244.

55. Mathiesen, E. (1933).

56. Sólo 1 , de las 26 empresas recogidas en el cuadro 10, Fuentes e hijos, no adopta la forma societaria.

57. Este capital medio se refiere sólo a las sociedades constituidas en la provincia de Jaén. Ver: Hernández, S. (1997), p. 513.

58. Para una caracterización del empresariado agrícola y aceitero, ver: Hernández, S. (1977), pp. 338-356. 
CUADRO 10

\section{CLASIFICACIÓN POR PROFESIONES Y ORIGEN GEOGRÁFICO \\ DE LOS SOCIOS DE EMPRESAS EXPORTADORAS DE ACEITE DE OLIVA EN ANDALUCÍA EN 1934}

\begin{tabular}{lrrlrr}
\hline Profesión & $\begin{array}{c}\text { Núm. } \\
\text { socios }\end{array}$ & $\begin{array}{r}\text { \% sobre } \\
\text { el total }\end{array}$ & $\begin{array}{l}\text { Lugar de } \\
\text { residencia }\end{array}$ & $\begin{array}{c}\text { Núm. } \\
\text { socios }\end{array}$ & $\begin{array}{r}\text { \% sobre } \\
\text { el total }\end{array}$ \\
\hline Propietario & 13 & 8,78 & Córdoba & 11 & 7,43 \\
Labrador & 1 & 0,68 & Jaén & 24 & 16,22 \\
Industrial & 5 & 3,38 & Málaga & 16 & 10,81 \\
Comercio & 66 & 44,59 & Sevilla & 51 & 34,46 \\
Banca & 1 & 0,68 & Total Andalucía & $\mathbf{1 0 2}$ & $\mathbf{6 8 , 9 2}$ \\
Abogado & 4 & 2,70 & Alameda & 1 & 0,68 \\
Ingeniero & 3 & 2,03 & Valencia & 1 & 0,68 \\
Militar & 1 & 0,68 & Alicante & 2 & 1,35 \\
Empleado & 1 & 0,68 & Madrid & 7 & 4,73 \\
Estudiante & 3 & 2,03 & Herencia & 5 & 3,38 \\
Sociedades & 3 & 2,03 & Barcelona & 1 & 0,68 \\
Sus labores & 3 & 2,03 & Tortosa & 4 & 2,70 \\
Menor & 13 & 8,78 & San Sebastián & 2 & 1,35 \\
No definida & 31 & 20,95 & Haro & 9 & 6,08 \\
Total & $\mathbf{1 4 8}$ & $\mathbf{1 0 0 , 0 0}$ & Extranjeros & 3 & 2,03 \\
& & & Otras regiones & $\mathbf{3 5}$ & $\mathbf{2 3 , 6 5}$ \\
& & & No definido & 11 & 7,43 \\
& & & Total & $\mathbf{1 4 8}$ & $\mathbf{1 0 0 , 0 0}$ \\
\hline
\end{tabular}

Fuente: L'Olivicultore, núm. 3, marzo de 1935, p. 37.

cio del siglo XX fuera la figura del "propietario» Ia que gozara de una mayor consideración, llevaría a muchos de estos socios a catalogarse como tales, situando en un lugar secundario los aspectos industriales y comerciales de su actividad empresarial ${ }^{59}$. Este sería el caso de muchos de los "propietarios» que nos aparecen en el cuerpo social de las empresas exportadora andaluzas ${ }^{60}$.

En cuanto al origen geográfico, presenta una problemática similar. Muchos de estos empresarios, a la hora de indicar su procedencia en las escrituras de constitución de la sociedad, harían referencia a su vecindad en ese momento y no a la original. Ahora bien, para calibrar el peso de otras regiones en el desarrollo de este sector

59. María Sierra apunta como en la Sevilla del siglo XIX las profesiones de comerciante e industrial eran consideradas con "con un cierto desprecio», consecuencia de una mentalidad en la que permanecen resistencias formales heredadas del Antiguo Régimen. Sierra, $\mathbf{M}^{\mathrm{a}}$ (1992), p. 30.

60. Los miembros de sociedades como Carbonell, S.C.; Hijos de Ibarra, S.A.; Riva Hermanos, S.A.; Larios, S.A., entre otras, de conocido origen mercantil o industrial, se registran en las escrituras de constitución como "propietarios». En el caso mejor conocido, la familia Carbonell, Castejón pone de manifiesto como estos empresarios "no distraen sus esfuerzos en otros campos, como la agricultura, ya que en algunos momentos en que adquieren fincas, se sirven de las mismas para sus fines industriales...». Castejón, R. (1977), p. 242. 
en Andalucía, no sólo hemos de tener en cuenta el número de empresarios, sino 1 importancia de su participación. En este sentido, el número de socios habría de s€ matizado con el número de empresas en las que éstos tuvieron responsabilidad y 1 envergadura de las mismas. De ambas consideraciones se deduce que la incidenci de este tipo de empresariado fue muy elevada. En una primera fase, con anterior dad a 1914, familias como los Ybarra o Carbonell, estarían en el origen de las em presas más importantes del sector. Con posterioridad a 1914, otros empresarios, e este caso catalanes, contribuirían a explicar el auge de esta actividad.

\section{CONCLUSIONES}

De lo dicho hasta el momento se desprende que, en el primer tercio del sigl XX, el sector aceitero español consiguió avances muy significativos que le per mitieron penetrar con fuerza en el mercado internacional. Para explicar estos lo gros hay que recurrir al incremento de la producción, a los precios competitivos a la calidad alcanzada por los aceites españoles. Sin embargo, no debe perders de vista el papel jugado por las empresas exportadoras, que pronto adoptaro] modelos de organización modernos, que les posibilitaron una mejor capitalizació $\mathrm{y}$, en casos como el noruego, el desarrollo de una eficaz política comercial.

Esta actividad exportadora estuvo controlada, mayoritariamente, por u empresariado de origen catalán. A medida que nuevos mercados se fueron abrien do, fundamentalmente en el continente americano, y que el aceite de la gran re gión productora, Andalucía, alcanzó niveles aceptables de calidad, muchos de es tos empresarios trasladaron su actividad a esta región, estableciendo vínculo societarios con propietarios, fabricantes de aceite, comerciantes, industrias, etc. d la propia Andalucía y de otras regiones de España. Lo que en un principio fueror sólo sucursales de empresas matrices localizadas en Barcelona, Tortosa o Reus, e1 poco tiempo se convirtieron en establecimientos importantes, situados en los puer tos y núcleos urbanos bien comunicados de la región andaluza. Estas empresa: canalizaban más del $70 \%$ de la exportación de aceite de oliva llevaba a cabo po empresas españolas, a mediados de los años treinta.

En el caso de Andalucía, el empresariado de esta actividad exportadora present: un perfil, tanto por su origen geográfico como por el sociológico, substancialment distinto a los del sector del cultivo y la fabricación del aceite. No fueron los grande: propietarios, a partir de empresas familiares, ni los medianos, a partir de socieda des tipo cooperativa, los que lideraron el sector del refino y la comercialización propiciando, de este modo, en un poceso de integración vertical del sector aceite ro ${ }^{61}$. Por su parte, los empresarios con origen en el comercio o en la actividac

61. Que conozcamos, sólo a comienzos de los años treinta, en plena crisis del sector aceitero, se plantearos los productores de aceite de oliva introducirse en la comercialización. La empresa que se creó para tal objetivo, li 
industrial que caracterizaban la exportación, muchos de ellos procedentes de otras regiones españolas y de otros países europeos, tampoco penetraron, de forma significativa, en el cultivo del olivar y la fabricación del aceite ${ }^{62}$. El resultado es que, al menos durante el primer tercio del siglo XX, una nítida frontera separaba ambos subsectores.

Independientemente del empresariado, en el proceso de expansión de las exportaciones españolas de aceite de oliva se puede apreciar una clara visión regional. A la vez que los aceites catalanes pierden cuota de mercado a favor de los andaluces, se registra una especialización de éstos en aceites de calidad, en gran medida destinados a encabezar las mezclas realizadas por los exportadores franceses e italianos. Por el contrario, la exportación andaluza, pese a la mejora de la calidad, tiene su mayor activo en la gran producción, que no deja de incrementarse.

\section{BIBLIOGRAFÍA}

ABREU FERNÁNDEZ, F. (1983), La formación de capital en la ría de Vigo, 1880-1940. El sector naval y la industria conservera. Tesis doctoral, Universidad de Santiago.

- (1998), «El caso del asentamiento de la fábrica de conservas de pescado de la empresa Masso en las dos últimas décadas del siglo pasado», en I Encuentro de Económica Aplicada, Barcelona, junio de 1998 .

BARRÓN GARCÍA, J.I. (1992), La economía de Cantabria en la etapa de la Restauración, 1875-1908. Santander.

CARMONA BADIA, X. (1983), Producción textil rural e actividades maritimo-pesqueiras na Galiza, 1750-1905, Tesis doctoral, Universidad de Santiago.

— (1985), «La industria conservera gallega. 1840-1905», en Papeles de Economía Española, pp. 177-191.

— (1990), "Crisis y transformación de la base industrial gallega, 1850-1936», en NADAL, J. y CATALÁN, J., Pautas regionales de la industrialización española (siglos XIX y XX). Barcelona, pp. 35-39.

- (1994), «Recursos, organización y tecnología en el crecimiento de la industria española de conservas de pescado, 1900-1936», en NADAL, J. y CATALÁN, J. (eds.), La cara oculta de la industrialización española. La modernización de los sectores no líderes (siglo XIX y $X X$ ), Madrid, pp. 127-162.

CASTEJÓN MONTIJANO, R. (1977), Génesis y desarrollo de una sociedad mercantil e industrial en Andalucía: La casa Carbonell de Córdoba (1866-1918), Córdoba.

CASTEJÓN MONTIJANO, R. y otros (1980), «Empresas y empresarios andaluces en el siglo XIX: una primera aproximación», en Cuadernos de Ciencias Económicasy Empresariales, núm. 6 , pp. 225-256.

"Cooperativa Nacional de Productores de Aceite de Oliva Puro", pese a contar con el apoyo de las más importantes asociaciones de olivareros, acabó fracasando. Ver Hernández, S. (1999b).

62. Para Castejón la falta de integración de los procesos industriales y comerciales sería un factor importante a la hora de explicar parte de los fracasos empresariales en Andalucía Castejón, R. (1977), p. 242. Este proceso si se produjo en otros subsectores de la industria agroalimentaria andaluza, caso de los vinos de Jerez. Ver Maldonado, J. (1999). 
DECROIX, J.L. (1964), Le developpement de l'industrie francçaise de la conserve, Parí D'AVIGNEU (1958), L'industrie des conserves de poisson en France metropolitaine, Rennes EICHENGREEN, G., IRWIN, D. (1996), «The Role of History in Biateral Trade Flows», e NBER, Working Paper, núm. 5.565.

F.E.A.O.E. (Federación Exportadores Aceite de Oliva de España). Sus fondos documentales está depositados en la Biblioteca de la Facultad de CC.EE. y EE. de la Universidad de Málaga

GAYA, M. (1930), «El aceite de oliva en Cataluña», en La industria aceitera, núm. 29, p. 11 HANN, W.S. (1948), «Observations on Fishprocessing in Norway», en Canner. Citado e. Industria conservera, marzo de 1949, pp. 16-17.

HERNÁNDEZ ARMENTEROS, S. (1997), El crecimiento económico en una región atrasada Jaén, 1850-1936. Tesis doctoral. Universidad de Granada.

- (1999a), «Viaje a la industria aceitera. La visita del noruego E. Mathiesen a las regione aceiteras españolas en 1933», en Revista de Estudios Regionales, en prensa.

- (1999b), «La búsqueda de la comercialización en el sector aceitero jiennense: la Coopera tiva Nacional de Productores de Aceite de Oliva Puro», en I Congreso de Cooperativism. Español, Osuna, 1999.

HILDEBRAND, K.G. (1981), «Los rasgos característicos de la industrialización de los paíse escandinavos y de Finlandia en el siglo XIX", en La industrialización europea. Estadios tipos, Barcelona.

HODNE, F. (1975), An Economic History of Norway, 1815-1970, Oslo.

GOULD, D.M. (1994), «Inmigran Links to the Home Country: Empirical Implications for U.S Bilateral Trade Flows», en Review of Economics and Statistics, núm. 76, pp. 302-316 INDUSTRIA CONSERVERA (1947), «Las conservas de pescado en Noruega», octubre-noviem bre de 1947, p. 10.

JACOBSSEN, N. (1953), «Situación de la economía noruega durante 1952», en Boletín Men sual de la Cámara de Comercio Hispano-Noruega, núm. 3, pp. 5-9.

JIMÉNEZ BLANCO, J.I. (1986), La producción agraria en Andalucía Oriental, 1874-1914 Madrid.

LINNEMANN, H. (1996), An Econometric Study of International Trade Flows. Amsterdam

LUNDE, G. (1933), «Om olivenolje. V. Om pávisning av raffinert olivenolje og sulfurolje vec fluorescensmálinger», en Tidsskrift for hermetikindustri, abril, 1933.

LUNDE, G., MATHIESEN, (1932), «II. Undersokelse av importet olivenolje av hosten 1929. 30 og 1930-31», en Tidsskrift for hermetikindustri, marzo de 1933.

LUNDE, G., MATHIESEN, E., MIKKELSEN, E. (1933), «Om olivenolje. VI. Undersokelse ar importert olivenolje av hosten 1932-33», en Tidsskrift for hermetikindustri, diciembre, 1933

MALDONADO, J. (1999a), «El sector vinícola en Andalucía», en Andalucía a debate. Terce. ras Jornadas "Industrialización y desindustrialización de Andalucía. Una revisiór. historiográfica», Granada, junio de 1999.

- (1999), «La industria vinícola, sus derivados y auxiliares en Andalucía (siglos XVIII-XIX)» en La industrialización andaluza. Un balance historiográfico de veinticinco años de inves. tigación. Seminario en homenaje a Jordi Nadal. Almería.

MATHIESEN, E. (1932), «I. Utvinning og undersokelsesmetoder», en Tidsskrift for hermetikindustri, marzo de 1932.

- (1933), «Studier over olivenolje pa produksjonsstedene i Spania og Frankrike», en Tidsskrifi for Hermetikindustri, febrero de 1933, pp. 51-58.

MILWARD, A. y SAUL, S.B. (1979), El desarrollo económico de la Europa continental. Madrid. 
NADAL, J. (1987), «La industria fabril española en 1900. Una aproximación», en NADAL, J., CARRERAS, A. y SUDRIA, C. La economía española en el siglo XX. Una perspectiva histórica, Barcelona, pp. 23-61.

OLIVICULTORE, L.: RIVISTA OLERIA ITALIANA (1929), Roma, Societá Nazionale degli Olicoltori.

PAREJO, A. y ZAMBRANA, Fco. (1994), «La modernización de la industria del aceite en España en los siglos XIX y XX», en NADAL, J. y CATALÁN, J., La cara oculta de la industrialización española, Madrid, pp. 13-42.

PEREIRA, E. (1943), «La evolución de una gran industria», en Industria conservera, agosto de 1943, pp. 8-9.

POLLARD, S. (1991), La conquista pacífica. La industrialización de Europa (1760-1970), Zaragoza.

RAMÓN MUÑOZ, R. (1997), «La industria aceitera española y los mercados exteriores: un análisis comparativo, 1852-1913», en Actas del II Encuentro de Historia Económica, Valencia.

(1998), «Estructura empresarial, empreses i canvi tècni en la indústria de l' oli d'oliva de les comarques de Lleida, 1890-1936. Inédito.

- (1999), «Patterns of Specialisation in the Intemational Market for Olive Olil before World War II: Evidence and Hypothese», en WILLIAMSON, J. y PAMUK, S. (eds.), Economic Change in the Mediterranean before 1950, Londres.

RIOS, S. (1999), «La industria conservera de Ayamonte: desde sus orígenes hasta la II República», en La industrialización andaluza. Un balance historiográfico de veinticinco años de investigación. Seminario en homenaje a Jordi Nadal. Almería.

ROMERO ATELA, T. (1996), «Los empresarios catalanes en Córdoba, 1800-1914», en Els catalans a Espanya, 1760-1914, Barcelona, 1996.

SAVASTANO, G. y SERINACO, E. (1935), «Di alcuni procesi moderni di raffinazione», en L'Olivicultore, núm. 8, agosto de 1935, pp. 3-16.

SENNER, P. (1943), «La industria de conservas en Noruega», en Industria conservera, octubre de 1943, pp. 4-5.

SIERRA, Mª (1992), La familia Ybarra, empresarios y políticos. Sevilla.

SIMPSON, J. (1997), La agricultura española (I765-1965): la larga siesta. Madrid.

ZAMBRANA, Fco. (1984), «El aceite de oliva y su dependencia del mercado internacional de las grasas vegetales. Un análisis histórico, 1861-1935», en Agricultura y Sociedad, Núm. 33.

- (1987), Crisis y modernización del olivar español. Madrid.

- (1999), «La industria del aceite de oliva en Andalucía durante el primer franquismo», en La industrialización andaluza. Un balance historiográfico de veinticinco años de investigación. Seminario en homenaje a Jordi Nadal. Almería. 